\title{
ACE2 receptor usage reveals variation in susceptibility to SARS-CoV and SARS-CoV-2 infection among bat species
}

\author{
Huan Yan ${ }^{1,5}$, Hengwu Jiao ${ }^{2,5}$, Qianyun Liu', Zhen Zhang1, Qing Xiong1, Bing-Jun Wang², Xin Wang1,

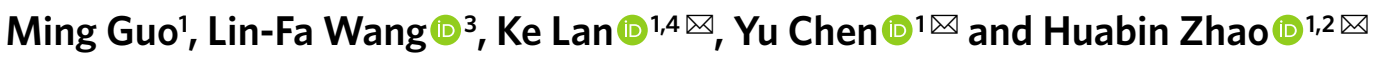

Bats are the suggested natural hosts for severe acute respiratory syndrome coronavirus (SARS-CoV) and the causal agent of the coronavirus disease 2019 (COVID-19) pandemic, SARS-CoV-2. The interaction of viral spike proteins with their host receptor angiotensin-converting enzyme 2 (ACE2) is a critical determinant of potential hosts and cross-species transmission. Here we use virus-host receptor binding and infection assays to examine 46 ACE2 orthologues from phylogenetically diverse bat species, including those in close and distant contact with humans. We found that 24, 21 and 16 of them failed to support infection by SARS-CoV, SARS-CoV-2 or both viruses, respectively. Furthermore, we confirmed that infection assays in human cells were consistent with those in two bat cell lines. Additionally, we used genetic and functional analyses to identify critical residues in bat ACE2 receptors associated with viral entry restrictions. Our results suggest that many bat species may not be the potential hosts of one or both viruses and that no correlation was identified between proximity to humans and probability of being natural hosts of SARS-CoV or SARS-CoV-2. This study demonstrates dramatic variation in susceptibility to SARS-CoV and SARS-CoV-2 infection among bat species and adds knowledge towards a better understanding of coronavirus-bat interaction.

T he unprecedented pandemic of coronavirus disease 2019 (COVID-19), caused by the new coronavirus SARS-CoV-2, has led to major threats to public health and economic development. It is therefore critically important to identify natural or intermediate hosts of SARS-CoV-2 to prevent further spread of COVID-19 and future emergence of similar diseases. Inferred from sequence similarity of human and bat virus genomes, it was suggested that horseshoe bats (Rhinolophus spp.) might be natural hosts of SARS-CoV and SARS-CoV-2 (refs. ${ }^{1-3}$ ). These suggestions have resulted in misguided fears and unwarranted attacks on many bats-including species other than Rhinolophus-thereby seriously impacting efforts towards bat conservation ${ }^{4}$. Given the remarkable diversity of bats, which includes more than 1,400 species across the globe ${ }^{5}$, assessing the possibility that diverse bat species act as potential hosts of SARS-CoV and SARS-CoV-2 is urgent and crucial for both controlling outbreaks and protecting populations of wildlife.

Angiotensin-converting enzyme 2 (ACE2) is the main host cell receptor of SARS-CoV and SARS-CoV-2 and plays a vital role in mediating viral entry to cause infection ${ }^{1,6}$. The interaction of a virus with its host receptor has been repeatedly demonstrated to serve as a primary determinant of host range ${ }^{7,8}$. In this study, we tested ACE2 orthologues from 46 bat species across the phylogeny, including species occurring in urban and in rural areas, for their ability to support the entry of SARS-CoV and SARS-CoV-2 using virus-host receptor binding and infection assays. Hence, this study assessed whether diverse bat species are potential hosts of SARS-CoV or SARS-CoV-2. Moreover, by determining the correlation between proximity to humans and probability of being natural hosts of the two viruses, these results provide important insights into pandemic control and wildlife conservation.

\section{Results}

Evolution of ACE2 in bats inhabiting urban or rural areas. We collected ACE2 orthologues from 46 bat species across the phylogeny (Fig. 1 and Supplementary Table 1). These species contained 28 species that roost or forage in urban areas near humans and 18 species more restricted to rural areas and hence likely to have minimal contact with humans (Supplementary Table 2). In total, we examined 46 species representing 11 bat families (Supplementary Table 3). After aligning the protein sequences of bat ACE2 orthologues, we examined 25 critical residues involved in the binding of the surface spike glycoprotein (S protein) of SARS-CoV-2 (ref. ${ }^{9}$ ) (Extended Data Fig. 1). Genetic variations were observed in nearly all these 25 sites, which may have led to different abilities to support entry of SARS-CoV and SARS-CoV-2 (ref. ${ }^{9}$ ). Furthermore, we detected at least 22 amino acid sites that are putatively under positive selection (Supplementary Table 4), which is indicative of heterogeneous selection pressure across sites. Notably, four of these positively selected sites are in the binding region of ACE2 to the SARS-CoV-2 S protein (Supplementary Table 4).

Interaction between bat ACE2 orthologues and SARS-CoV or SARS-CoV-2 receptor binding domain. Efficient binding between the S protein and the ACE2 receptor is essential for SARS-CoV and SARS-CoV-2 entry. This binding is mainly mediated by the interaction between the critical residues on the receptor-binding domain

'State Key Laboratory of Virology, Modern Virology Research Center, College of Life Sciences, Wuhan University, Wuhan, China. ${ }^{2}$ Department of Ecology, Tibetan Centre for Ecology and Conservation at WHU-TU, Hubei Key Laboratory of Cell Homeostasis, College of Life Sciences, Wuhan University, Wuhan, China. ${ }^{3}$ Duke-NUS Medical School, Singapore, Singapore. ${ }^{4}$ Frontier Science Center for Immunology and Metabolism, Wuhan University, Wuhan, China. 5These authors contributed equally: Huan Yan, Hengwu Jiao.凶e-mail: klan@whu.edu.cn; chenyu@whu.edu.cn; huabinzhao@whu.edu.cn 


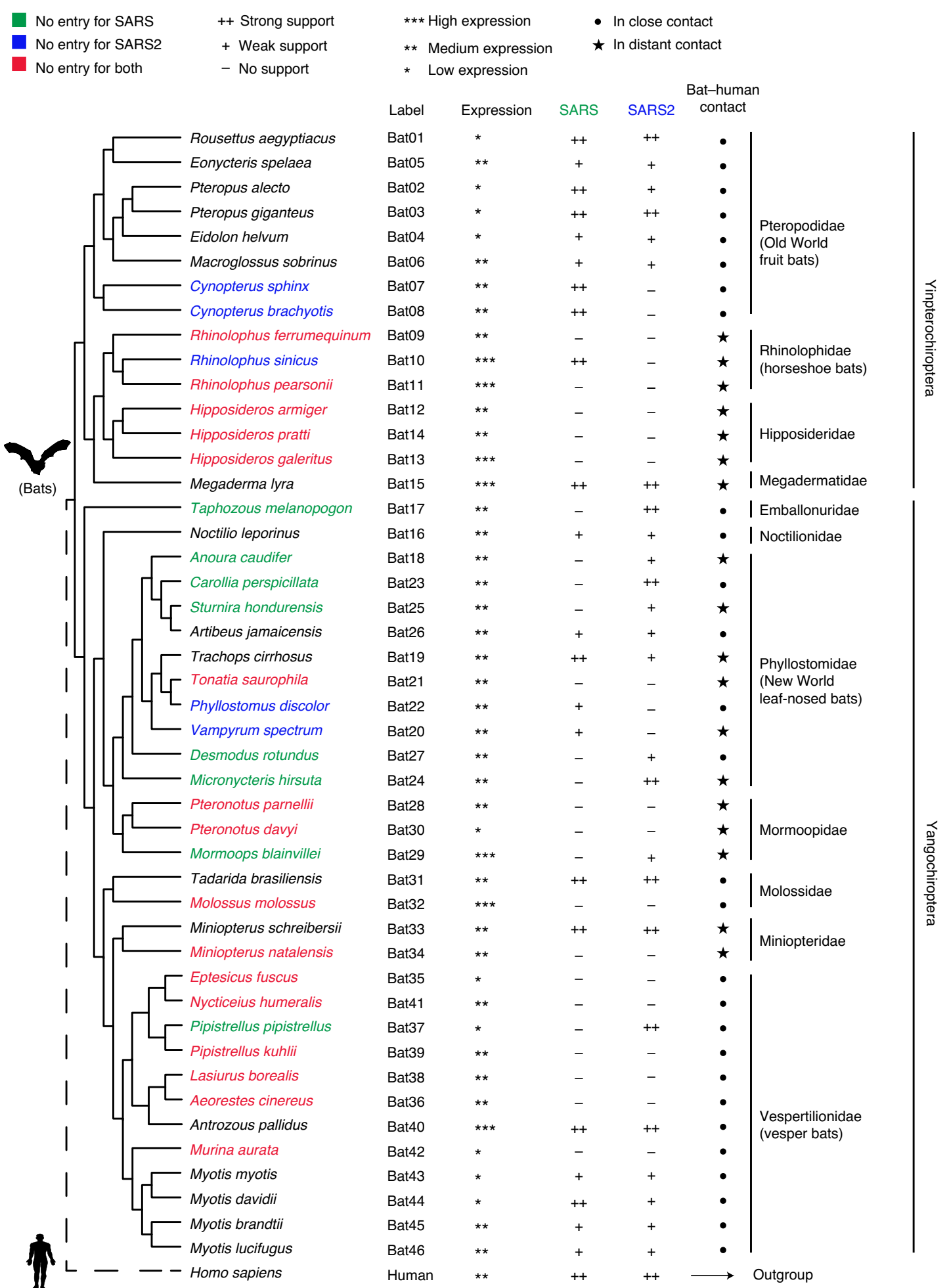

Fig. 1 | Phylogenetic tree of $\mathbf{4 6}$ bat species in this study. The labels of bat species in our experiments are indicated. Expression levels determined by western blot (Fig. 2a) are shown with asterisk symbols compared with human ACE2: the triple asterisk indicates high expression, the double asterisk indicates medium expression and the single asterisk indicates low but detectable expression. The ability of bat ACE2 to support SARS-CoV and SARS-CoV-2 pseudovirus entry is shown with different signs (Fig. 3a,b): infection data are presented as percentage mean values of bat ACE2 supporting infection compared with the infection supported by human ACE2. Infection efficiency $<5 \%$ is indicated with a minus sign, between 5 and $50 \%$ with a plus sign and $>50 \%$ with a double plus sign. Bat phylogeny was taken from previous studies ${ }^{28-30}$.

(RBD) and ACE2. To characterize the receptor function of ACE2 orthologues in a range of diverse bat species, we generated a stable cell library consisting of cell lines expressing the respective 46 bat
ACE2 orthologues through lentiviral transduction of $293 \mathrm{~T}$ cells lacking ACE2 expression ${ }^{10}$. All bat ACE2 orthologues were exogenously expressed at a comparable level after puromycin selection, 
a

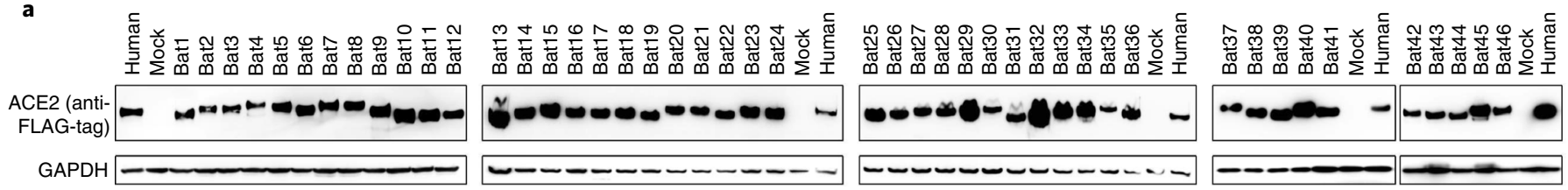

b
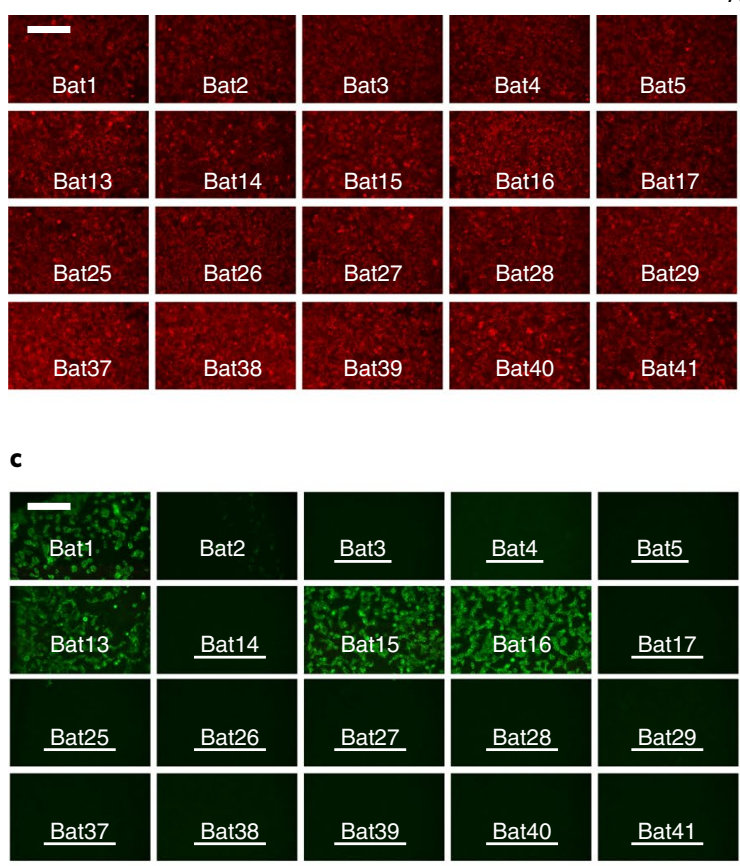

d

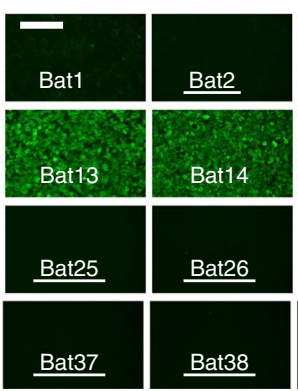

ACE2 expression (anti-FLAG-tag)

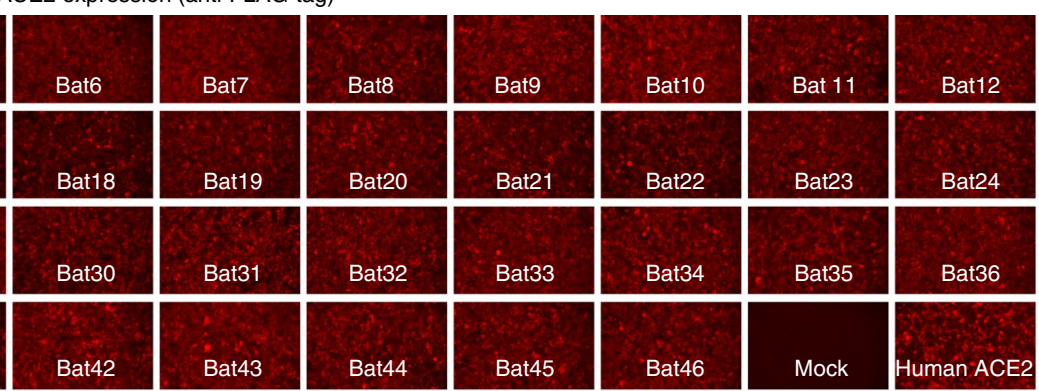

SARS-RBD-hFc binding

\begin{tabular}{|l|l|l|}
\hline Bat5 & Bat6 & Bat7 \\
\hline Bat17 & Bat18 & Bat19 \\
\hline Bat29 & Bat30 & Bat31 \\
\hline Bat41 & Bat42 & Bat43 \\
\hline
\end{tabular}

SARS2-RBD-hFc binding

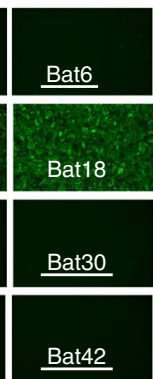

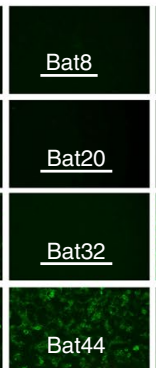
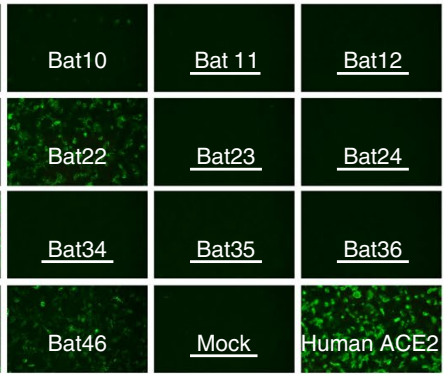

Fig. 2 | Expression of bat ACE2 orthologues and their interaction with the SARS-CoV and SARS-CoV-2 RBD. a, Western blot detected the expression levels of ACE2 orthologues on 293T stable cells by targeting the C-terminal 3XFLAG-tag. Glyceraldehyde 3-phosphate dehydrogenase was employed as a

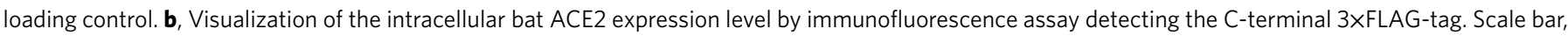
$100 \mu \mathrm{m}$. c,d, Assessment of the interaction between different ACE2 orthologues and SARS-CoV-RBD-hFc (c) or SARS-CoV-2-RBD-hFc (d) proteins. Species that do not support efficient binding are underlined. $293 \mathrm{~T}$ cells stably expressing the different bat $\mathrm{ACE} 2$ orthologues were incubated with $5 \mu \mathrm{g} \mathrm{ml}^{-1}$ of the recombinant proteins at $37^{\circ} \mathrm{C}$ for $1 \mathrm{~h}$; binding efficiency was examined by Alexa Fluor 488 goat anti-human IgG via fluorescence assay. Scale bar, $200 \mu \mathrm{m}$.

as indicated by western blot and immunofluorescence assays detecting the C-terminal 3×FLAG-tag (Fig. 2a,b).

To analyse the interaction, we produced recombinant SARS-CoV or SARS-CoV-2 RBD human immunoglobulin G (IgG) Fc fusion proteins (RBD-hFc), previously reported to be sufficient to bind human ACE2 efficiently ${ }^{11,12}$. Protein binding efficiency was tested on the bat ACE2 cell library by means of immunofluorescence or flow cytometry targeting the human Fc. As expected, binding was almost undetectable on mock $293 \mathrm{~T}$ cells but a strong binding signal was detected in the $293 \mathrm{~T}$ cells expressing human ACE2 (Fig. 2c,d). Consistent with previous reports ${ }^{13,14}$, SARS-CoV-2 RBD showed higher binding to human ACE2 than SARS-CoV, which can also be observed on many bat ACE2 orthologues (Fig. 2c,d). Previous reports have shown that only a small fraction of ACE2 orthologues from tested mammalian species could not bind with SARS-CoV-2 S protein $\left(n=6\right.$ of 49 species $^{7} ; n=5$ of 17 species $\left.^{15}\right)$. However, our study revealed that many bat species ( $n=32$ and $n=28$ of 46 species) do not support efficient binding with SARS-CoV-RBD and SARS-CoV-2-RBD, respectively (Fig. 2c,d). The overall profiles of bat ACE2 to bind to SARS-CoV and SARS-CoV-2 RBD are generally comparable; a few showed contrasting modes of binding preferences (Fig. 2c,d). For instance, Bat22 could bind to SARS-CoV 

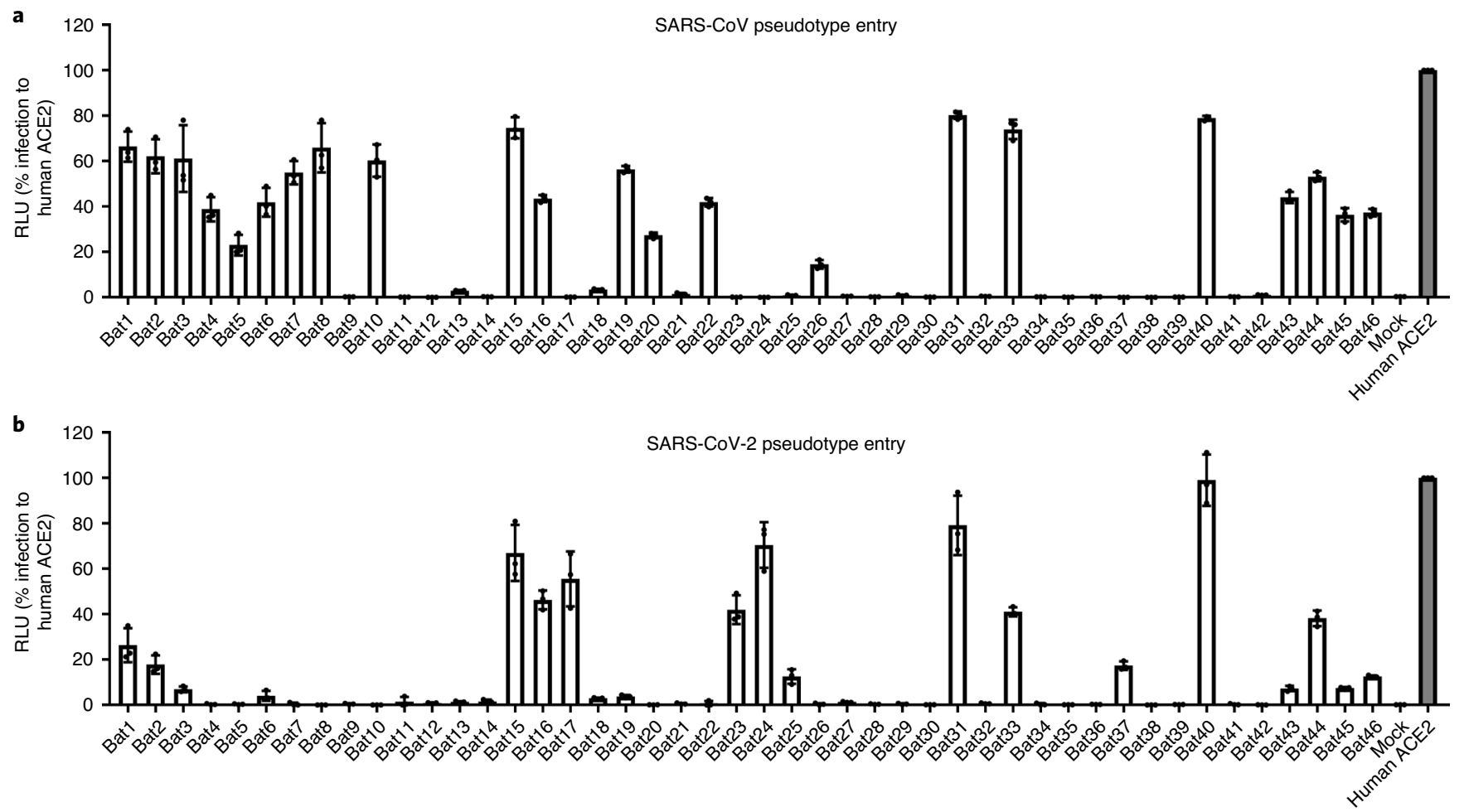

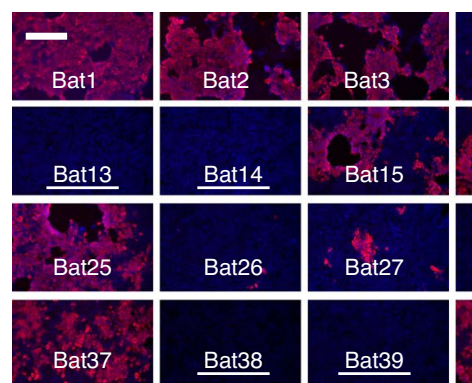

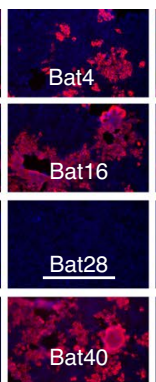

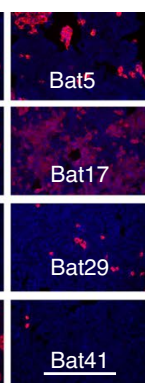

SARS-CoV-2 infection $-\mathrm{N}$ protein
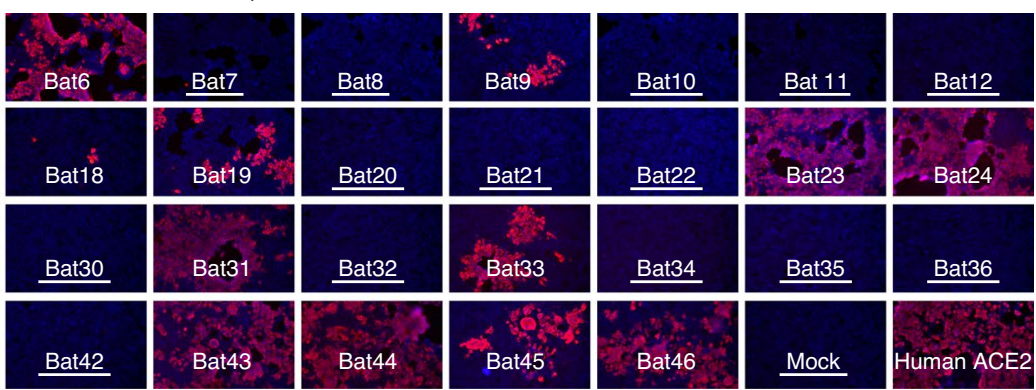

Fig. 3 | Characterization of bat ACE2 orthologues mediating entry of SARS-CoV and SARS-CoV-2 viruses. a,b, Ability of bat ACE2 orthologues to support the entry of SARS-CoV and SARS-CoV-2 pseudovirus. 293T cells expressing bat ACE2 orthologues in a 96-well plate were infected with VSV-dG-Luc pseudotyped with SARS-CoV (a) and SARS-CoV-2 (b) spike proteins, respectively. Intracellular luciferase activity was determined at $20 \mathrm{~h}$ post-infection.

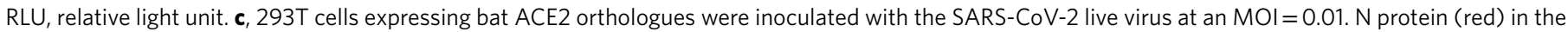

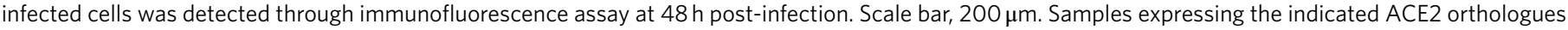
that showed almost no entry for SARS-CoV-2 live virus are underlined. Data shown are representative results from 3 independent experiments and are presented as the mean $\pm \mathrm{s} . \mathrm{d}$. ( $n=3$ for $\mathbf{a}$ and $n=2$ for $\mathbf{b})$.

but not SARS-CoV-2, whereas Bat14, 21 and 40 could bind to SARS-CoV-2 but not SARS-CoV (Fig. 2c,d). Flow cytometry analysis showed consistent results (Extended Data Fig. 2).

Overall, the RBD-hFc binding assays demonstrated that bat ACE2 orthologues showed different affinity and selectivity levels to SARS-CoV and SARS-CoV-2, indicating that the ACE2 receptors of many bat species may not support efficient SARS-CoV and SARS-CoV-2 infection.

Receptor function of bat ACE2 orthologues to support the entry of SARS-CoV and SARS-CoV-2 using pseudotyped and live viruses. To further evaluate the receptor function of different bat ACE2 orthologues, we employed a vesicular stomatitis virus (VSV)-based rhabdoviral pseudotyping system to mimic the coronavirus spike protein-mediated single-round entry ${ }^{15}$. SARS-CoV and SARS-CoV-2 pseudotypes were generated by assembling the coronavirus spike proteins and replication-deficient VSV with the VSV glycoprotein gene replaced with a fluorescence protein (VSV-dG-GFP) or a firefly luciferase (VSV-dG-Luc) reporter ${ }^{15}$. Both viruses showed minimal background infection on 293T cells, but efficient infection on 293T-human ACE2 cells (Extended Data Fig. 3). The susceptibility of the 293T cells expressing bat ACE2 orthologues was then examined with SARS-CoV and SARS-CoV-2 pseudotypes. The results showed that bat ACE2 orthologues have varying abilities to support coronavirus entry and different preferences for SARS-CoV and SARS-CoV-2. (Fig. 3a,b and Extended Data Fig. 4). Pseudotypes with green fluorescent protein (GFP) reporter showed similar results (Extended Data Fig. 5). Notably, we found that 24, 21 and 16 of the 46 bat species showed almost no entry for SARS-CoV, SARS-CoV-2 and both viruses, respectively (Figs. 1 and 3a,b and Supplementary Table 5), suggesting that these species are not likely to be potential hosts of either or both coronaviruses. The bat species showing no viral entry include those that occur in urban areas and those more restricted to rural areas 
(Fig. 1), suggesting that there is no correlation between proximity to humans and probability of being natural hosts of SARS-CoV or SARS-CoV-2. Although horseshoe bats were suggested as potential natural hosts of SARS-CoV and SARS-CoV-2 (refs. ${ }^{1-3}$ ), only one of the three species examined (Rhinolophus sinicus) supported SARS-CoV entry; this species was suggested as the potential host of SARS-CoV ${ }^{3,16}$. None of these tested horseshoe bats showed entry for SARS-CoV-2 (Figs. 1 and 3). These results unambiguously indicate that ACE2 receptor usage is species-dependent.

The SARS-CoV-2 S protein used in this study for pseudotyping contains a D614G mutation, which is currently a dominant variation ${ }^{17}$. The D614G mutation remarkably improved the in vitro infectivity of SARS-CoV-2 but may not significantly affect the receptor interaction since it is not in the $\mathrm{RBD}^{18}$. Indeed, we identified a very similar susceptibility profile using an original strain without D614G (Extended Data Fig. 4). We further demonstrated that the pseudotyped entry assay mimics the entry of live viruses through a SARS-CoV-2 infection assay (Fig. 3c). As expected, the profile of SARS-CoV-2 N protein expression is highly consistent with the results from the VSV-dG-based pseudotyped virus entry assay, except for some ACE2 that showed relatively higher infection efficiency (for example, Bat43-46) compared with the pseudovirus infection assay, which may be attributed to the different virus strains used (Fig. 3c). In addition, the live virus infection resulted in the phenotype of plaque formation, while the pseudotypes showed evenly distributed, single-round infection (Extended Data Fig. 5), which also partially explains why some bat ACE2 showed higher infection in the live virus infection assay.

When comparing the RBD-hFc binding and pseudotyped entry profiles, we found that binding and susceptibility are not always consistent, although the phenotypes were reproducible. For instance, some species (Bat12, 13, 14) were able to bind to SARS-CoV-2 RBD-hFc efficiently but could not support infection of the same virus, indicating that high binding affinity does not guarantee efficient viral entry (Figs. 2 and 3). In contrast, some species (Bat3-8) were defective or less efficient in SARS-CoV RBD-hFc binding but supported the entry of the same virus to some degree (Figs. 2 and 3). We hypothesize that such minimal binding may be sufficient for viral entry mediated by those ACE2 orthologues; alternatively, additional residues outside the traditional $\mathrm{RBD}$ region might be required for efficient interaction. These hypotheses should be tested in the future. Together, our results demonstrated dramatic variation of susceptibility to SARS-CoV and SARS-CoV-2 infection among bat species, suggesting that SARS-CoV and SARS-CoV-2 can selectively use some bat ACE2 as functional receptors for viral entry and many-if not most-bat ACE2 are not favoured by one or both viruses.

Evaluation of critical residues in bat ACE2 orthologues affecting viral binding and entry efficiency or specificity. We comprehensively analysed the relationship between critical RBD binding sites in bat ACE2 sequences and their ability to support SARS-CoV and SARS-CoV-2 RBD binding and viral entry. Several critical residues were identified that may play critical roles in the determination of species specificity (Extended Data Fig. 1). According to the sequence alignment, two species pairs (Bat33 and Bat34 and Bat 38 and Bat40) were selected to demonstrate the role of critical residues in RBD binding and viral entry because they were phylogenetically close but showed contrasting phenotypes for supporting RBD binding and viral entry. Specifically, Bat34 and Bat38 do not support SARS-CoV and SARS-CoV-2 RBD binding and infection, while Bat33 supports efficient binding and infection of both viruses and Bat40 supports infection of both viruses and to a lesser degree SARS-RBD binding (Figs. 2 and 3). We compared their protein sequences and highlighted the residues that may affect RBD interaction. For example, substitutions I27K, N31G and K42E were observed when comparing Bat33 with Bat34, while Q24L, E30K, K35Q and G354N were present between Bat38 and Bat40 (Fig. 4a). We hypothesized that the discrepancy in binding and infection phenotype is determined by their differences in critical residues for RBD interaction. To test this hypothesis, we designed a residue swap mutagenesis assay to investigate the role of critical residues on RBD binding and virus entry (Fig. 4a). We generated four swap mutations and corresponding 293T stable cell lines to test whether these substitutions could achieve gain-of-function and loss-of-function. All bat ACE2 orthologues and related mutants were expressed at a comparable level after lentiviral transduction, as indicated by the immunofluorescence of the C-terminal 3×FLAG-tag (Fig. 4b). Recombinant SARS-CoV and SARS-CoV-2 RBD-hFc proteins were applied to the cells expressing different ACE2 and binding efficiency was evaluated by immunofluorescence (Fig. 4c) and flow cytometry assays (Fig. 4d). As expected, the swap of critical residues on the selected four bat ACE2 changed their receptor function to the opposite, except for Bat38 mutant, which remained unable to bind SARS-CoV RBD-hFc (Fig. 4c,d). GFP (Fig. 4e) and luciferase levels (Fig. 4f) from the pseudotyped virus entry assay and the $\mathrm{N}$ protein staining from the live SARS-CoV-2 infection assay (Fig. 4g) further confirmed our hypothesis at the viral entry level. Structure modelling of bat ACE2/SARS-CoV-2-RBD complexes showed that the substitutions of I27K and N31G between Bat33 and Bat34 lead to a reduced packing interaction and the substitution of $\mathrm{K} 42 \mathrm{E}$ disrupts the hydrogen bond with Y449, which may be related to the difference of susceptibility between Bat33 and Bat34 (Fig. 4h,i and Extended Data Fig. 6). In comparison, the substitutions of Q24L and $\mathrm{E} 30 \mathrm{~K}$ between Bat38 and Bat40 destroyed the favourable hydrophilic interactions with N487 and K417, respectively (Extended Data Fig. 6).

In addition, two bat cell lines, the lung epithelial cell line $\mathrm{Tb} 1$ $\mathrm{Lu}$ of Tadarida brasiliensis (Bat31) and the kidney epithelial cell line of Pteropus alecto (Bat2), were used to validate our findings derived from human HEK293T cells. Endogenous ACE2 expression

Fig. 4 | Evaluation of the critical binding sites determining the species-specific restriction of SARS-CoV and SARS-CoV-2 binding and entry. a, Swap mutagenesis assay to investigate the role of critical residues on bat ACE2 orthologues for tropism determination. Residues involved in RBD (according to the structure between SARS2-RBD and human ACE2, Protein Data Bank 6MOJ) interaction are shown in the table. Residues that changed in the mutagenesis assay are marked in red. $\mathbf{b}$, The expression level of the bat ACE2 orthologues and related mutants in transduced 293T cells was determined by an immunofluorescence assay recognizing the 3xFLAG-tag. Scale bar, $200 \mu \mathrm{m}$. c,d, Binding efficiency of SARS2-RBD-hFc and SARS2-RBD-hFc on 293T cells expressing bat ACE2 and related mutants. Cells were incubated with $5 \mu \mathrm{g} \mathrm{ml} l^{-1}$ of recombinant proteins at $37^{\circ} \mathrm{C}$ for $1 \mathrm{~h}$ and then washed and incubated with a secondary antibody recognizing human Fc. Immunostaining (c) and flow cytometry (d) were conducted to show binding efficiency. Scale bar, $200 \mu \mathrm{m}$. e,f, Ability of the indicated ACE2 and related mutants to support the entry of coronavirus pseudotypes. The 293T cells expressing the indicated ACE2 and their mutants were infected with SARS-CoV and SARS-CoV-2 pseudotypes expressing GFP (e) and luciferase (f). Infection was analysed at $20 \mathrm{~h}$ post-infection. Scale bar, $200 \mu \mathrm{m}$. Data are presented as the mean with s.d. $(n=2) . \mathbf{g}, 293 \mathrm{~T}$ cells infected by the SARS-CoV-2 live virus at an MOI=0.01; the infection was examined at $48 \mathrm{~h}$ post-infection through $\mathrm{N}$ protein (red) immunostaining. Nuclei were stained with Hoechst 33342 (blue). Scale bar, $200 \mu \mathrm{m}$. $\mathbf{h}, \mathbf{i}$, Comparison of the interface between Bat33/SARS-CoV-2-RBD and Bat34/SARS-CoV-2-RBD. Bat33 and its complexed RBD are coloured cyan and gold, respectively (h); Bat34 and its complexed RBD are coloured wheat and green, respectively (i). The mutated residues in ACE2 and the corresponding residues in SARS-CoV-2-RBD are shown and labelled. The red dotted lines between residues indicate hydrogen or ionic bonds. 
was almost undetectable in these two cell lines, accounting for at least 1,000 folds lower than the susceptible Vero-E6 cells (Extended Data Fig. 7a). Therefore, these cells cannot support the entry of SARS-CoV and SARS-CoV-2. We successfully generated Tb $1 \mathrm{Lu}$ stable cell lines expressing human ACE2 and bat ACE2 (Bat2, 3,
$31,32)$ since the transduction efficiency of $\mathrm{Tb} 1 \mathrm{Lu}$ is much higher than that of PakiT03 cells (Extended Data Fig. 7b). As expected, Tu $1 \mathrm{Lu}$ were susceptible to both SARS-CoV and SARS-CoV-2 when human ACE2 or some bat ACE2 orthologues (Bat2, 3 and 31) were expressed, yet remained non-susceptible when an ACE2 of a closely

a

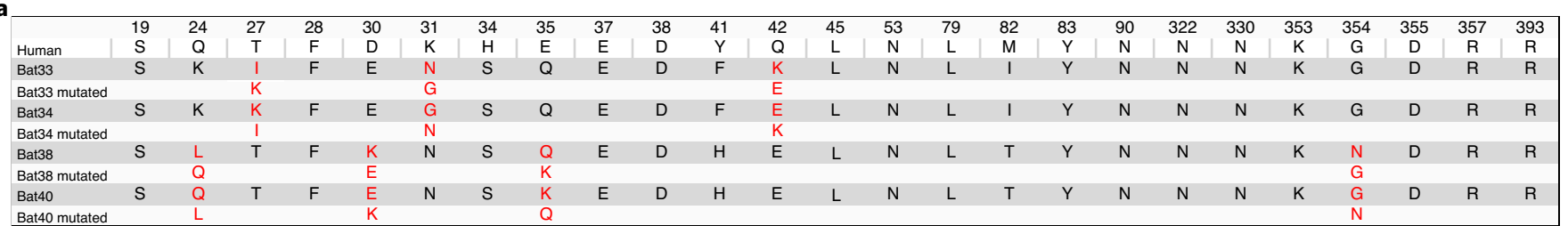

b
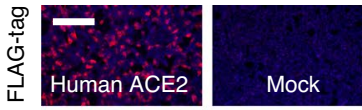
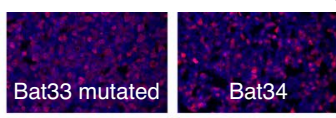

SARS RBD binding
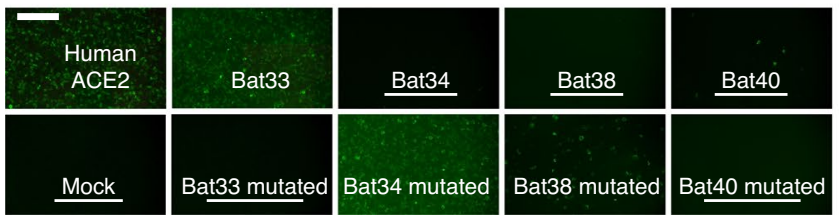

SARS RBD binding
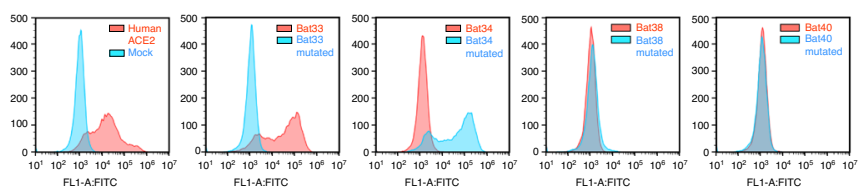

e

SARS-CoV pseudovirus entry
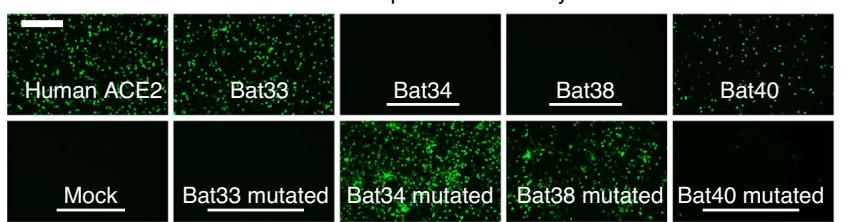
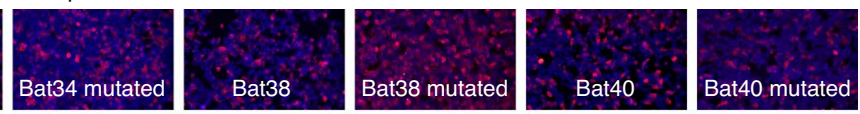

SARS2 RBD binding
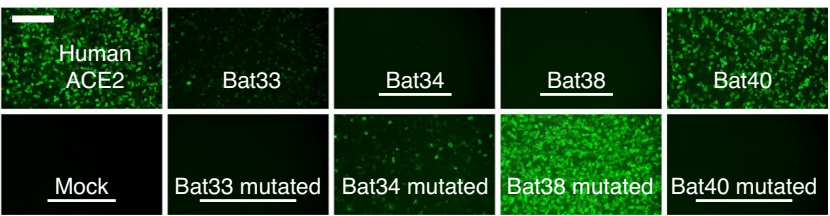

SARS-CoV pseudovirus entry

SARS-CoV-2 pseudovirus entry
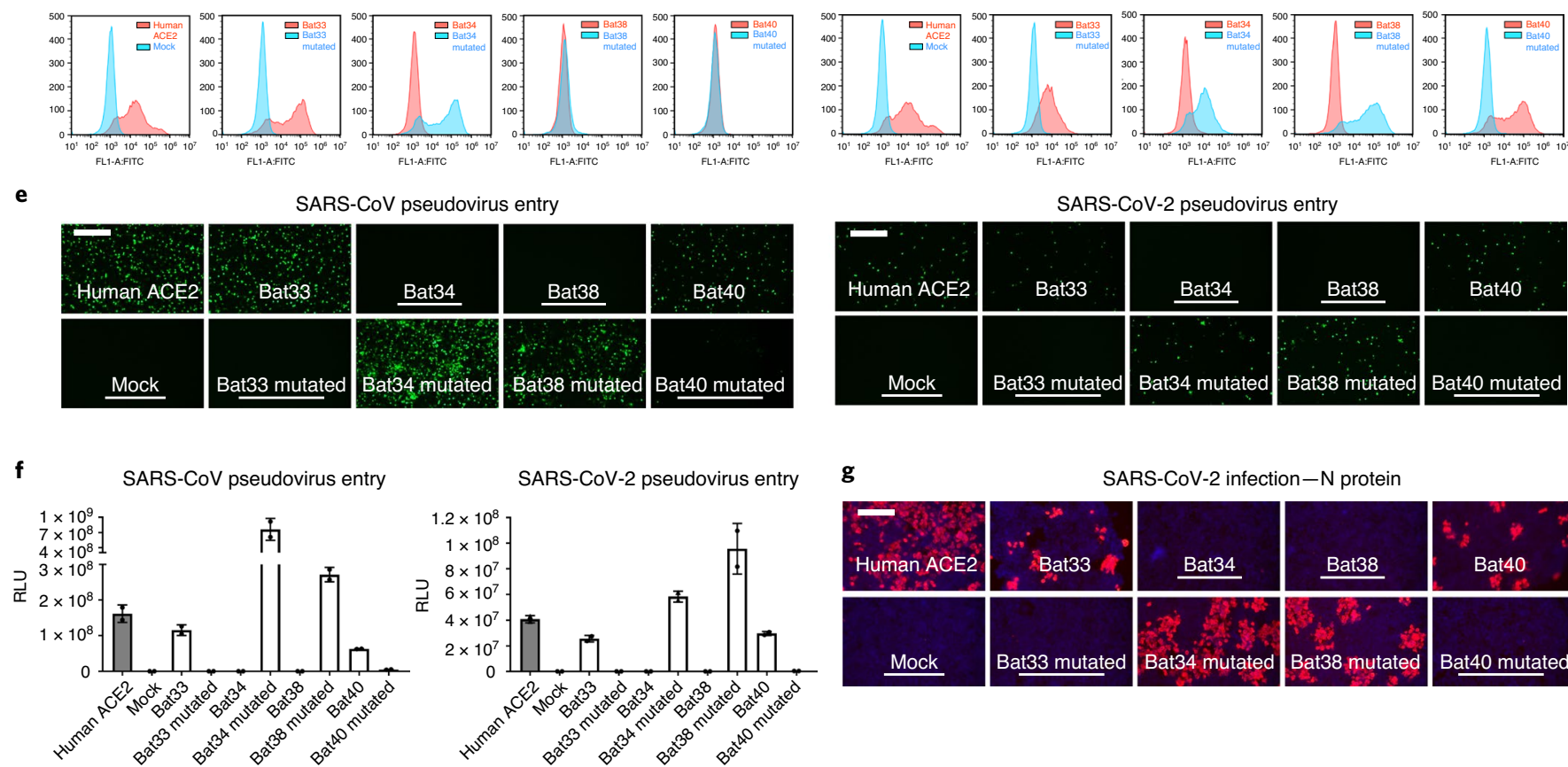

SARS-CoV-2 pseudovirus entry
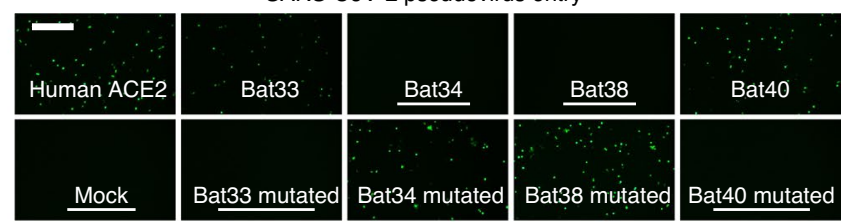

h

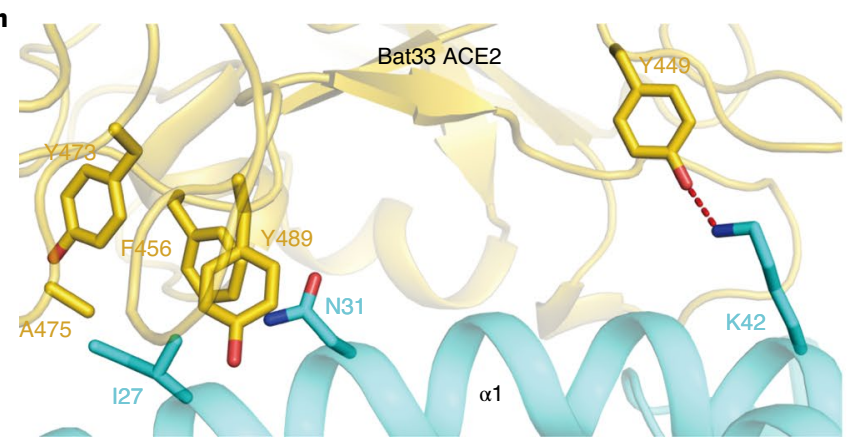

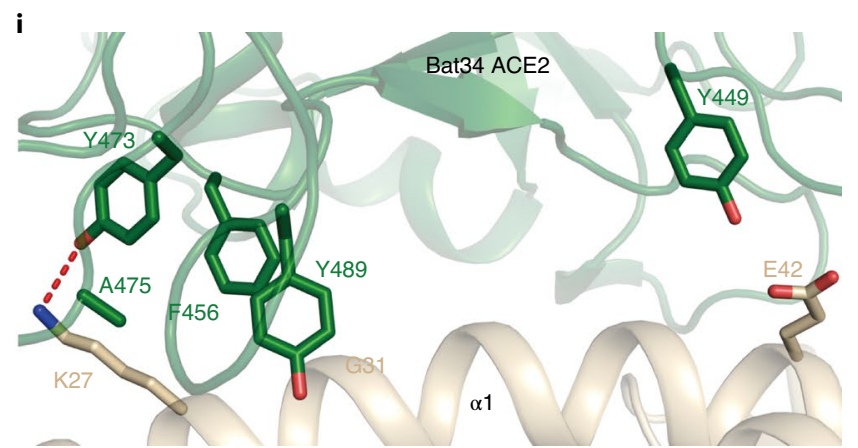


related species (Bat32) was expressed (Extended Data Fig. 7c-e). Furthermore, we conducted SARS-CoV and SARS-CoV-2 pseudovirus entry assays on the two bat cell lines transiently transfected with various bat ACE2 (Bat2, 3, 31, 32, 33, 34, 38, 40) and their mutants (mutant Bat33, 34, 38 and $40 \mathrm{~m}$ ). The results were consistent with those derived from human cells, further confirming that ACE2 is the main receptor for the species-specific entry of SARS-CoV and SARS-CoV-2 in these bat cells (Extended Data Fig. 7f,g).

\section{Discussion}

Our study provides genetic and functional evidence from ACE2 receptor usage to show dramatic variation of susceptibility to SARS-CoV and SARS-CoV-2 infection among bat species. Our sampling covers representative species from 11 bat families, hence providing a broad picture of bat phylogeny. Moreover, our study included 28 species inhabiting urban areas and 18 species that are not common in cities or do not roost in buildings. Our functional assays demonstrated that there is no correlation between proximity to humans and probability of being natural hosts of SARS-CoV or SARS-CoV-2. Our results are only partially consistent with a recently published prediction based on sequence similarity, which estimated a binding score between ACE2 and the SARS-CoV-2 S protein for each vertebrate species ${ }^{9}$. The predicted binding scores for all 37 bat species fell into low $(n=8)$ and very low $(n=29)$ categories ${ }^{9}$, suggesting that all examined bat species are at low risk for SARS-CoV-2 infection. Our study included 36 of the 37 previously examined bat species (Fig. 1 and Extended Data Fig. 1); 21 of these appeared to support SARS-CoV-2 entry by their ACE2 receptors (Figs. 1 and 3), strongly suggesting that these bats are at risk for SARS-CoV-2 infection. These disparities between in silico analyses and functional experiments unambiguously suggest the importance of experimental data for confirmation of in silico analyses since our understanding of ACE2 sequences and structures is incomplete thus far. We note that our functional experiments also have limitations. For instance, it would be interesting to test bat ACE2 function in their respective species-specific cells if all bat cell lines were available. It is also important to note that the lack of viral infection in bat cell lines without ACE2 expression cannot rule out the possibility that alternative receptors are expressed in other cell types. Moreover, there were also some inconsistencies for some bat ACE2 between binding and infection. These inconsistencies may have resulted from some binding events that are defective in triggering the correct conformational change required for downstream entry $^{13,19}$. In addition, unlike binding, which is a dynamic process ${ }^{20}$, entry is irreversible and can accumulate over time. Thus, we should be cautious about predicting the ability of ACE2 orthologues to support entry only based on their binding affinities to viral RBDs. Our genetic and functional evidence revealed critical residues of bat ACE2 that are involved in supporting SARS-CoV-2 entry (Fig. 4). However, these residues are not the genetic determinant of New World monkey ACE2 orthologues mediating SARS-CoV-2 entry $^{7}$ and many bat ACE2 orthologues carrying residues that were considered unfavourable in the same study (H41 and E42) ${ }^{7}$ were fully functional in our study (Fig. 4), further confirming the complexity of ACE2 functionality.

We found that closely related species can show strikingly different ACE2 receptor usage. For example, Rhinolophus sinicus can support SARS-CoV entry, whereas its congeneric relatives Rhinolophus ferrumequinum and Rhinolophus pearsonii cannot (Figs. 1 and 3), even though some polymorphic sites of ACE2 may have occurred in Rhinolophus sinicus populations ${ }^{21}$. These findings clearly show that ACE2 receptor usage is species-dependent. Accordingly, although some bats might be potential hosts of ancestral SARS-CoV and SARS-CoV-2 (refs. ${ }^{1-3}$ ), one cannot assume that all bat species or individuals can carry these viruses. Although certain bat species are frequently observed to carry coronaviruses closely related to human viruses in terms of sequence similarity ${ }^{22}$, there is no solid and direct evidence showing the initial spillover from bats to humans and other animals. Nevertheless, humans infected with coronavirus should maintain distance from bats that can use ACE2 as a viral receptor because many bat species are endangered and may be susceptible to human coronaviruses ${ }^{23}$, as suggested for many other mammals ${ }^{9,24}$. Indeed, the International Union for Conservation of Nature has assessed that over one-third of bat species are threatened or data-deficient and over half of all bat species have unknown or decreasing population trends ${ }^{25}$. Thus, bats need protection more than ever. Our study supports the calls that public education on bat biology will reduce the threat to bats ${ }^{4,25}$. In fact, all bats are potentially safe as long as they are treated with care and respect. We should work collaboratively to combat the pandemic and identify which species are potential hosts and not fear those species that are not hosts of the virus. Instead, we must respect and care for those species that are potential hosts and learn about the impact of human activities on their natural habitats, which may lead to zoonotic spillover events.

\section{Methods}

ACE2 sequence acquisition and selective pressure analysis. We obtained 46 full-length coding sequences of bat ACE2 in this study, of which 32 were taken from a recent study ${ }^{9}$ and 14 were newly extracted from published or recently sequenced genome assemblies (see Supplementary Table 1 for the sources and accession numbers for the sequences and assemblies). Next, we aligned the deduced ACE2 protein sequences using the MUSCLE program (v.3.8.31) ${ }^{26}$ (see Extended Data Fig. 1 for the resulting alignment). The sequence logo was generated with WebLogo (https://weblogo.berkeley.edu/logo.cgi). We performed selective pressure analysis on bat ACE2 using CodeML implemented in PAML (v.4.9a ${ }^{27}$. Two comparisons of site models (M1a and M2a, M8a and M8) were used to predict positively selected sites ${ }^{27}$. The input tree was the species tree (Fig. 1) taken from previous studies ${ }^{28-30}$.

Cell culture. HEK293T cells (293T, catalogue no. CRL-3216; ATCC) and VERO E6 cells (catalogue no. CRL-1586; ATCC) were cultured in DMEM (Gibco) supplemented with $10 \%$ fetal bovine serum (FBS), $2.0 \mathrm{mM}$ of L-glutamine, $110 \mathrm{mgl}^{-1}$ of sodium pyruvate and $4.5 \mathrm{gl}^{-1}$ of $\mathrm{D}$-glucose. The lung epithelial bat cell line Tb $1 \mathrm{Lu}$ (catalogue no. CCL-88; ATCC) and an I1-Hybridoma (catalogue no. CRL-2700; ATCC) cell line secreting a monoclonal antibody targeted against the VSV glycoprotein were cultured in MEM with Earle's balanced salts and $2.0 \mathrm{mM}$ of L-glutamine and 10\% FBS. The kidney epithelial bat cell line PakiT03 was a generous gift from L.-F. Wang and was cultured in DMEM:F-12 (Gibco) supplemented with $10 \%$ FBS. All cells were cultured at $37^{\circ} \mathrm{C}$ in $5 \% \mathrm{CO}_{2}$ with the regular passage of every $2-3 \mathrm{~d}$. 293T stable cell lines overexpressing ACE2 orthologues were maintained in growth medium supplemented with $1 \mu \mathrm{g} \mathrm{ml}^{-1}$ of puromycin.

Plasmids. Human codon-optimized complementary DNA sequences encoding various ACE2 orthologues and their mutants fused with a C-terminal $3 \times$ FLAG-tag (DYKDHD-G-DYKDHD-I-DYKDDDDK) were commercially synthesized and subcloned into a lentiviral transfer vector (pLVX-IRES-puro) through the EcoRI and NotI restriction sites. The DNA sequences of human codon-optimized SARS-CoV S protein (CUHK-W1, GenBank AY278554.2) and SARS-CoV-2 S protein (Wuhan-Hu-1, GenBank MN908947) were amplified from the plasmids pCMV/hygro-SARS-CoV-S (VG40150-G-N; Sino Biological) and pCAGGS-SARS-CoV-2-S-c9 (gifted by W. Li, National Institute of Biological Science, Beijing, China) into the pCAGGS vector with a C-terminal 18-amino acid deletion to improve VSV pseudotyping efficiency ${ }^{31,32}$. The D614G mutation was introduced into the SARS-CoV-2-S coding sequence for higher in vitro infection efficiency. The plasmids for the expression of coronavirus RBD-IgG Fc fusion proteins were generated by inserting the coding sequences of SARS-CoV RBD (amino acids 318-516) and SARS-CoV-2 RBD (amino acids 331-530) into the pCAGGS vector to express fusion proteins with C-terminal human Fc (IgG1) and an N-terminal CD5 secretion leading sequence (MPMGSLQPLATLYLLGMLVASVL).

Generation of ACE2 stable expression cell lines. 293T and Tb $1 \mathrm{Lu}$ cells overexpressing ACE2 orthologues were generated by lentiviral transduction. Specifically, the lentivirus was produced by cotransfection of lentiviral transfer vector carrying ACE2 coding sequences (pLVX-EF1a-Puro; from GENEWIZ) and packaging plasmids pMD2G (plasmid no. 12259; Addgene) and psPAX2 (plasmid no. 12260; Addgene) into 293T cells through Lipofectamine 3000 (Thermo Fisher Scientific). The lentivirus-containing supernatant was collected and pooled at 24 and $48 \mathrm{~h}$ post-transfection. $293 \mathrm{~T}$ and $\mathrm{Tb} 1 \mathrm{Lu}$ cells were transduced by the 
lentivirus (1:1 dilution of culture medium and lentivirus-containing supernatant) after $16 \mathrm{~h}$ in the presence of $8 \mu \mathrm{g} \mathrm{ml}^{-1}$ polybrene. Stable cells expressing various ACE2 orthologues were selected and maintained in growth medium with puromycin $\left(1 \mu \mathrm{g} \mathrm{ml}^{-1}\right)$. Cells selected for at least $10 \mathrm{~d}$ were considered as stable cell lines and used in different experiments.

Immunofluorescence assay to evaluate the expression levels of ACE2 orthologues. The expression levels of ACE2 orthologues were evaluated by an immunofluorescence assay used to detect the C-terminal 3XFLAG-tags. The 293T or $\mathrm{Tb} 1 \mathrm{Lu}$ cells used for the analysis were seeded in the poly-lysine-pretreated 96-well plate at a cell density of $5 \times 10^{5}$ per $\mathrm{ml}(100 \mu \mathrm{l}$ per well) and cultured for $24 \mathrm{~h}$. Cells were fixed with $4 \%$ paraformaldehyde at room temperature for $10 \mathrm{~min}$, permeabilized with $0.2 \%$ Triton X-100/PBS at room temperature for $10 \mathrm{~min}$ and blocked with $1 \% \mathrm{BSA}$ at $37^{\circ} \mathrm{C}$ for $30 \mathrm{~min}$. Next, they were incubated with a mouse monoclonal antibody targeting the FLAG-tag (9A3, catalogue no. 8146S; Cell Signaling Technology) diluted in $1 \% \mathrm{BSA} / \mathrm{PBS}$ at $37^{\circ} \mathrm{C}$ for $1 \mathrm{~h}$. After three rounds of PBS washing, cells were incubated with $2 \mu \mathrm{g} \mathrm{ml}^{-1}$ of the Alexa Fluor 594-conjugated goat anti-rabbit IgG (catalogue no. A11032; Thermo Fisher Scientific) diluted in $1 \%$ BSA /PBS at room temperature for $30 \mathrm{~min}$. The nucleus was stained blue with Hoechst 33342 (1:5,000 dilution in PBS). Images were captured with a fluorescence microscope (MI52-N; Mshot).

\section{Production of VSV reporter viruses pseudotyped with coronavirus spike} proteins. Coronavirus spike protein pseudotyped viruses (CoV-psV) were packaged according to a previously described protocol using a replicate-deficient VSV-based rhabdoviral pseudotyping system (VSV-dG) ${ }^{33}$. The VSV-G glycoprotein-deficient VSV exogenously expressing enhanced GFP (VSV-dG-GFP) or firefly luciferase (VSV-dG-Luc) were rescued by a reverse genetics system from Kerafast. To produce CoV-psV, Vero E6 cells were transfected with the plasmids overexpressing SARS2-CoV (pCAGGS-SARS-S-dc) and SARS2-CoV-2 spike proteins (pCAGGS-SARS2-S-dc) through the Lipofectamine 3000 reagent. After $36 \mathrm{~h}$, the transfected cells were transduced with VSV-dG reporter viruses diluted in serum-free Opti-MEM for $1 \mathrm{~h}$ at $37^{\circ} \mathrm{C}$ (at a genome equivalent $=100$ ). Transduced cells were washed with culture medium once and then replenished with fresh culture medium with I1-hybridoma-cultured supernatant containing anti-VSV monoclonal antibody (1:100 dilution) to neutralize the infectivity of the residual input viruses. The $\mathrm{CoV}$-psV-containing supernatants were collected at $24 \mathrm{~h}$ after transduction, clarified at 12,000 r.p.m. for $2 \mathrm{~min}$ at $4{ }^{\circ} \mathrm{C}$ and immediately transferred to $-80^{\circ} \mathrm{C}$ for storage. The viral titre (genome equivalents) was determined by quantitative PCR with reverse transcription (RT-qPCR). The RNA copies in the virus-containing supernatant were detected using the VSV-L gene sequences (Supplementary Table 6).

Pseudotype entry assay. The 293T or bat lung epithelial ( $\mathrm{Tb} 1 \mathrm{Lu}$ ) stable cell lines overexpressing various ACE2 orthologues were trypsinized and resuspended with SARS-CoV or SARS-CoV-2 pseudotyped viruses (at a genome equivalent $=100$ ) in DMEM with $10 \%$ FBS. Next, they were seeded at $5 \times 10^{4}$ in a well of a 96 -well plate to allow attachment and viral infection simultaneously. At $16-24 \mathrm{~h}$ after infection, images of infected cells with GFP expression were acquired with a fluorescence microscope (MI52-N). Intracellular luciferase activity was determined by a Bright-Glo Luciferase Assay Kit (Promega Corporation) and measured with a SpectraMax iD3 Multi-well Luminometer (Molecular Devices) or a GloMax 20/20 Luminometer (Promega Corporation). Alternatively, the PakiT03 bat cell line or Tb 1 Lu cells were transfected with plasmids overexpressing various ACE2 orthologues (based on the pLVX-IRES-puro vector) by Lipofectamine 3000 reagent and infected with the SARS-CoV or SARS-CoV-2 pseudotyped viruses carrying luciferase (at a genome equivalent $=100$ ) at $24 \mathrm{~h}$ post-transfection. Cells were lysed using $1 \times$ Bright-Glo luciferase assay reagent and determined using a GloMax 20/20 Luminometer.

Coronavirus RBD-hFc binding assay. Recombinant SARS-CoV-RBD-hFc and SARS-CoV-2-RBD-hFc proteins were produced by transient transfection of 293T cells with Lipofectamine 3000. Transfected cells were cultured in FreeStyle 293 Expression Medium (Thermo Fisher Scientific) and the supernatants containing the recombinant proteins were collected at 2 and $4 \mathrm{~d}$ post-transfection The RBD-hFc protein concentration was determined by comparing the target protein band with BSA standard dilutions through Coomassie Brilliant Blue staining. The RBD-hFc protein-containing supernatant $\left(5 \mu \mathrm{g} \mathrm{ml}^{-1}\right)$ was incubated with the 293T stable cell lines overexpressing different ACE2 orthologues for $1 \mathrm{~h}$ at $37^{\circ} \mathrm{C}$. Cells were washed twice with DMEM and then incubated with $2 \mu \mathrm{g} \mathrm{ml}^{-1}$ of Alexa Fluor 488-conjugated goat anti-human IgG (catalogue no. A11013; Thermo Fisher Scientific) diluted in DMEM with $2 \% \mathrm{FBS}$ for $30 \mathrm{~min}$ at $37^{\circ} \mathrm{C}$. For immunostaining, cells were washed twice with PBS. Images were captured with a fluorescence microscope (MI52-N). For the flow cytometry analysis, cells were detached by $5 \mathrm{mM}$ of EDTA/PBS and analysed with a CytoFLEX Flow Cytometer (Beckman Coulter Life Sciences).

SARS-CoV-2 live virus infection assay. The SARS-CoV-2 (strain IVCAS 6.7512) was provided by the National Virus Resource, Wuhan Institute of
Virology, Chinese Academy of Sciences. All SARS-CoV-2 live virus-related experiments were approved by the Level 3 Biosafety Committee (ABSL-3) of Wuhan University. All experiments involving SARS-CoV-2 were performed in the BSL-3 facility. SARS-CoV-2 was amplified on Vero E6 cells and stored at $-150^{\circ} \mathrm{C}$; the titre was determined on Vero E6 cells through a plaque assay. 293T cells expressing ACE2 orthologues were seeded on a poly-lysine-coated 96-well plate for $24 \mathrm{~h}$ before inoculation. Cells were infected with SARS-CoV-2 at an MOI $=0.01$ and then incubated in DMEM with $2 \%$ FBS for $48 \mathrm{~h}$ before testing. Cells were fixed with $4 \%$ paraformaldehyde in PBS at room temperature for $1 \mathrm{~h}$, permeabilized with $0.2 \%$ Triton X-100 for $10 \mathrm{~min}$ and then blocked with $1 \% \mathrm{BSA} / \mathrm{PBS}$ at $37^{\circ} \mathrm{C}$ for $1 \mathrm{~h}$. Cells were subsequently incubated with a mouse monoclonal antibody, SARS-CoV/SARS-CoV-2 Nucleocapsid Antibody (catalogue no. 40143-MM05; Sino Biological), at 1:500 dilution at $37^{\circ} \mathrm{C}$ for $1 \mathrm{~h}$ and then incubated with $2 \mu \mathrm{g} \mathrm{ml}^{-1}$ of Alexa Fluor 594-conjugated goat anti-mouse IgG antibody (catalogue no. A-11032; Thermo Fisher Scientific) at $37^{\circ} \mathrm{C}$ for $1 \mathrm{~h}$. The nucleus was stained with Hoechst 33342. Images were acquired with a fluorescence microscope (MI52-N).

Quantification of the endogenous expression of ACE2 orthologues by RT-qPCR. Endogenous ACE2 expression levels of Vero E6 (from Chlorocebus sabaeus), PakiT03 (from P. alecto, Bat2) and Tb $1 \mathrm{Lu}$ (from T. Brasiliensis, Bat31) cells were determined by RT-qPCR. In general, total RNA from cells cultured for $24 \mathrm{~h}$ was extracted by RNA-Easy Isolation Reagent (catalogue no. R701-02; Vazyme). cDNA was reverse-transcribed from $1 \mu \mathrm{g}$ of total RNA by the HiScript II 1st Strand cDNA Synthesis Kit (catalogue no. R212-02; Vazyme); $1 / 20$ volume of the cDNA was used as the template for the QPCR assay using the ChamQ Universal SYBR qPCR Master Mix (catalogue no. Q311-02; Vazyme) and a CFX96 Touch Real-Time PCR instrument (Bio-Rad Laboratories). Small nuclear ribonucleoprotein $\mathrm{D} 3$ polypeptide was used as an internal control for the normalization of the ACE2 relative expression level, which is presented as percentage expression compared with Vero E6 cell. Data are presented as the mean \pm s.e.m. $(n=3)$.

Homology-based structural modelling. Molecular models of different bat ACE2 were predicted by iterative threading assembly refinement (I-TASSER) v.5.1 (ref. ${ }^{34}$ ). Starting from the amino acid sequences, the I-TASSER algorithm constructed the full-length three-dimensional atomic models by structural template identification, followed by template-based fragment assembly simulations. The structure of SARS-CoV-2-RBD was obtained from the previously reported crystal structure of the human ACE2/SARS-CoV-2-RBD complex (Protein Data Bank $6 \mathrm{LZG})^{35}$. The docking of ACE2 and SARS-CoV-2-RBD was performed using RosettaDock (v.4.0) ${ }^{36}$. The binding energy of the ACE2/RBD complex was calculated using the InterfaceAnalyzer from the RosettaDock package and is represented in Rosetta energy units ${ }^{37}$. All types of non-covalent interactions were analysed using RING v.2.0 (ref. ${ }^{38}$ ) and LigPlot+ v.2.2 (ref. ${ }^{39}$ ). Structural alignment and visualization were implemented in PyMOL (v.2.5.0a0) $)^{40}$.

Statistical analysis. Data are expressed as mean values with s.d. unless otherwise stated. All experiments were repeated 3-5 times, each yielding similar results.

Reporting Summary. Further information on research design is available in the Nature Research Reporting Summary linked to this article.

\section{Data availability}

All data are available in the manuscript or supporting materials.

Received: 13 October 2020; Accepted: 29 January 2021; Published online: 1 March 2021

\section{References}

1. Zhou, P. et al. A pneumonia outbreak associated with a new coronavirus of probable bat origin. Nature 579, 270-273 (2020).

2. $\mathrm{Li}, \mathrm{W}$. et al. Bats are natural reservoirs of SARS-like coronaviruses. Science 310, 676-679 (2005).

3. $\mathrm{Hu}, \mathrm{B}$. et al. Discovery of a rich gene pool of bat SARS-related coronaviruses provides new insights into the origin of SARS coronavirus. PLoS Pathog. 13, e1006698 (2017)

4. Zhao, H. COVID-19 drives new threat to bats in China. Science 367, 1436 (2020).

5. Wilson, D. E. \& Mittermeier, R. A. Handbook of the Mammals of the World. Vol. 9: Bats (Lynx, 2009).

6. $\mathrm{Li}, \mathrm{W}$. et al. Angiotensin-converting enzyme 2 is a functional receptor for the SARS coronavirus. Nature 426, 450-454 (2003).

7. Liu, Y. et al. Functional and genetic analysis of viral receptor ACE2 orthologs reveals broad potential host range of SARS-CoV-2. Preprint at https://www. biorxiv.org/content/10.1101/2020.04.22.046565v2 (2020).

8. Liu, K. et al. Cross-species recognition of SARS-CoV-2 to bat ACE2. Proc. Natl Acad. Sci. USA 118, e2020216118 (2021). 
9. Damas, J. et al. Broad host range of SARS-CoV-2 predicted by comparative and structural analysis of ACE2 in vertebrates. Proc. Natl Acad. Sci. USA 117, 22311-22322 (2020).

10. Mossel, E. C. et al. Exogenous ACE2 expression allows refractory cell lines to support severe acute respiratory syndrome coronavirus replication. J. Virol. 79, 3846-3850 (2005).

11. Wong, S. K., Li, W., Moore, M. J., Choe, H. \& Farzan, M. A 193-amino acid fragment of the SARS coronavirus S protein efficiently binds angiotensin-converting enzyme 2. J. Biol. Chem. 279, 3197-3201 (2004).

12. Tai, W. et al. Characterization of the receptor-binding domain (RBD) of 2019 novel coronavirus: implication for development of RBD protein as a viral attachment inhibitor and vaccine. Cell. Mol. Immunol. 17, 613-620 (2020)

13. Wrapp, D. et al. Cryo-EM structure of the $2019-\mathrm{nCoV}$ spike in the prefusion conformation. Science 367, 1260-1263 (2020).

14. Shang, J. et al. Structural basis of receptor recognition by SARS-CoV-2. Nature 581, 221-224 (2020).

15. Nie, J. et al. Establishment and validation of a pseudovirus neutralization assay for SARS-CoV-2. Emerg. Microbes Infect. 9, 680-686 (2020).

16. Lau, S. K. P. et al. Severe acute respiratory syndrome coronavirus-like virus in Chinese horseshoe bats. Proc. Natl Acad. Sci. USA 102, 14040-14045 (2005).

17. Korber, B. et al. Tracking changes in SARS-CoV-2 spike: evidence that D614G increases infectivity of the COVID-19 virus. Cell 182, 812-827.e19 (2020).

18. $\mathrm{Hu}$, J. et al. The D614G mutation of SARS-CoV-2 spike protein enhances viral infectivity and decreases neutralization sensitivity to individual convalescent sera. Preprint at bioRxiv https://doi. org/10.1101/2020.06.20.161323 (2020).

19. Zhou, T. et al. Cryo-EM structures of SARS-CoV-2 spike without and with ACE2 reveal a $\mathrm{pH}$-dependent switch to mediate endosomal positioning of receptor-binding domains. Cell Host Microbe 28, 867-879.e5 (2020).

20. Jarmoskaite, I., AlSadhan, I., Vaidyanathan, P. P. \& Herschlag, D. How to measure and evaluate binding affinities. eLife 9, e57264 (2020).

21. Guo, H. et al. Evolutionary arms race between virus and host drives genetic diversity in bat severe acute respiratory syndrome-related related coronavirus spike genes. J. Virol. 94, e00902-20 (2020).

22. Banerjee, A., Kulcsar, K., Misra, V., Frieman, M. \& Mossman, K. Bats and coronaviruses. Viruses 11, 41 (2019).

23. Olival, K. J. et al. Possibility for reverse zoonotic transmission of SARS-CoV-2 to free-ranging wildlife: a case study of bats. PLoS Pathog. 16, e1008758 (2020)

24. Shi, J. et al. Susceptibility of ferrets, cats, dogs, and other domesticated animals to SARS-coronavirus 2. Science 368, 1016-1020 (2020).

25. Frick, W. F., Kingston, T. \& Flanders, J. A review of the major threats and challenges to global bat conservation. Ann. N. Y. Acad. Sci. 1469, 5-25 (2020).

26. Edgar, R. C. MUSCLE: multiple sequence alignment with high accuracy and high throughput. Nucleic Acids Res. 32, 1792-1797 (2004).

27. Xu, B. \& Yang, Z. PAMLX: a graphical user interface for PAML. Mol. Biol. Evol. 30, 2723-2724 (2013).

28. Teeling, E. C. et al. A molecular phylogeny for bats illuminates biogeography and the fossil record. Science 307, 580-584 (2005).

29. Almeida, F. C., Simmons, N. B. \& Giannini, N. P. A species-level phylogeny of Old World fruit bats with a new higher-level classification of the family Pteropodidae. Am. Mus. Novit. 3950, 1-24 (2020).

30. Rojas, D., Warsi, O. M. \& Dávalos, L. M. Bats (Chiroptera: Noctilionoidea) challenge a recent origin of extant neotropical diversity. Syst. Biol. 65, 432-448 (2016).

31. Fukushi, S. et al. Vesicular stomatitis virus pseudotyped with severe acute respiratory syndrome coronavirus spike protein. J. Gen. Virol. 86 2269-2274 (2005).
32. Schwegmann-Weßels, C. et al. Comparison of vesicular stomatitis virus pseudotyped with the $\mathrm{S}$ proteins from a porcine and a human coronavirus. J. Gen. Virol. 90, 1724-1729 (2009).

33. Whitt, M. A. Generation of VSV pseudotypes using recombinant $\Delta \mathrm{G}$-VSV for studies on virus entry, identification of entry inhibitors, and immune responses to vaccines. J. Virol. Methods 169, 365-374 (2010).

34. Yang, J. et al. The I-TASSER Suite: protein structure and function prediction. Nat. Methods 12, 7-8 (2015).

35. Wang, Q. et al. Structural and functional basis of SARS-CoV-2 entry by using human ACE2. Cell 181, 894-904.e9 (2020).

36. Gray, J. J. et al. Protein-protein docking with simultaneous optimization of rigid-body displacement and side-chain conformations. J. Mol. Biol. 331, 281-299 (2003)

37. Stranges, P. B. \& Kuhlman, B. A comparison of successful and failed protein interface designs highlights the challenges of designing buried hydrogen bonds. Protein Sci. 22, 74-82 (2013).

38. Piovesan, D., Minervini, G. \& Tosatto, S. C. E. The RING 2.0 web server for high quality residue interaction networks. Nucleic Acids Res. 44, W367-W374 (2016)

39. Laskowski, R. A. \& Swindells, M. B. LigPlot+: multiple ligand-protein interaction diagrams for drug discovery. J. Chem. Inf. Model. 51, 2778-2786 (2011).

40. Yuan, S., Chan, H. C. S., Filipek, S. \& Vogel, H. PyMOL and Inkscape bridge the data and the data visualization. Structure 24, 2041-2042 (2016).

\section{Acknowledgements}

We thank B. Fenton, L. Moretoo and D. M. Morales-Martínez for sharing their knowledge as to whether certain bats roost or forage in cities, E. C. Teeling for valuable comments, H. A. Lewin and J. Damas for sharing the ACE2 alignment, P. Zhou for providing the PakiT03 cells, Z.-L. Shi for providing the SARS-CoV-2 virus and M. Dai, Z. Huang, Y. Rao, J. Zhang and B. Wang from the ABSL-3 Laboratory of Wuhan University for their technical support. We thank the Beijing Taikang Yicai Foundation for supporting this work. This study was supported by a Special Fund for COVID-19 Research of Wuhan University, National Natural Science Foundation of China grants nos. 31722051 and 32041007 and National Science and Technology Major Project grant nos. 2018YFA0900801 and 2018ZX10733403.

\section{Author contributions}

H.Z., H.Y., Y.C. and K.L. designed study. H.Z., H.Y. and H.J. wrote the manuscript. H.Y., H.J., Q.L., Z.Z., Q.X., X.W. and M.G. performed the experiments. H.Y., H.Z., H.J., B.-J.W. L.-F. W. and Y.C. analysed the data.

\section{Competing interests}

The authors declare no competing interests.

\section{Additional information}

Extended data is available for this paper at https://doi.org/10.1038/s41559-021-01407-1. Supplementary information The online version contains supplementary material available at https://doi.org/10.1038/s41559-021-01407-1.

Correspondence and requests for materials should be addressed to K.L., Y.C. or H.Z. Peer review information Nature Ecology \& Evolution thanks Youchun Wang, Arinjay Banerjee and the other, anonymous, reviewer(s) for their contribution to the peer review of this work. Peer reviewer reports available.

Reprints and permissions information is available at www.nature.com/reprints.

Publisher's note Springer Nature remains neutral with regard to jurisdictional claims in published maps and institutional affiliations.

(c) The Author(s), under exclusive licence to Springer Nature Limited 2021 


\section{ACE2 orthologs}

Homo_sapien

Bat01 Rousettus_aegyptiacus

Bat02 Pteropus_alecto

Bat03 Pteropus_giganteus

Bat04 Eidolon_helvum.

Bat05 Eonycteris_spelaea

Bat06 Macroglossus_sobrinus

Bat07 Cymopterus_sphinx

Bat08 Cynopterus_brachyotis

Bat09 Rhinolophus_ferrumequinum

Bat10 Rhinolophus_sinicus

Bat11 Rhinolophus pearsonii

Bat12 Hipposideros_armiger

Bat13 Hipposideros_galeritus

Bat14 Hipposideros_pratti

Bat15 Megaderma_lyra

Bat16 Noctilio leporinus

Bat17 Taphozous_melanopogon

Bat18 moura_caudifer

Bat19 Trachops_cirrhosus

Bat20 vampyrum_spectrum

Bat21 Tonatia_saurophila Bat22 Phyllostomus discolor Bat23 Carollia_perspicillata Bat24 Micronycteris_hirsuta Bat25 Sturnira_hondurensis Bat26 Artibeus_jamaicensis Bat27 Desmodus rotundus Bat28 Pteronotus_parnellii Bat29 Pteronotus davyi Bat30 Mormoops_blainvillei Bat31 Tadarida_brasiliensis Bat32 Molossus_molossus Bat33 Miniopterus_schreibersii Bat34 Miniopterus_natalensis Bat35 Eptesicus_fuscus Bat36 Lasiurus borealis Bat37 Aeorestes_cinereus Bat38 Pipistrellus pipistrellus Bat39 Pipistrellus_kuhlii Bat40 Antrozous pallidus Bat41 Nycticeius_humeralis Bat42 Murina feae Bat43 Myotis_myotis Bat44 Mrotis davidi Bat45 Mrotis brandtii Bat46 Myotis_lucifugus

\section{Sequence Logo of \\ Bat ACE2 RBD binding region} (binding region

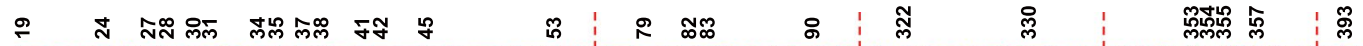
STIEEQAKTFL DKFNHEAE DLFYQS SL ASWNYNTIT STL AQMYPLQEIQNLT LPNMTQGFWENSMLT ANDL GKGDFRILM LRN STPEELAKTFLEKFNTEAEDLFYQS SL ASWDFNTNI I SKLAKTYQLDE IQDPE L PNMTETFWEKSVL T AM WL GKGDFR I M LRD

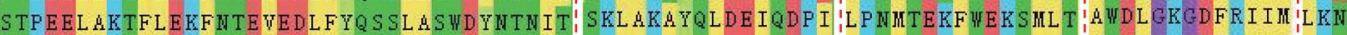

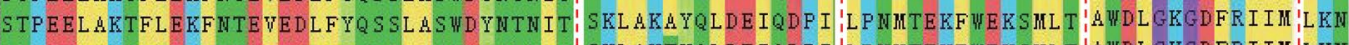
STPEEL AKTFLEKFNTEVEDLFYQS SL ASWDYNTM IT SKL AKTYQLDE IQDP I 'LPNMTEKFWEKSML T,AWDLGKGDFR I IM 'LKN STPEEL AKTFLEKFNTEAEDLFYQS SLASWDFNTMIT SKL AKTYRLDE IQDPE LPNMTEKFWEKSML T A HDLGKGFRI IM ILRN STPEELAKTFLEKFNTEVE DLFYE S SLASWDYNTNIT SKL AKNYQLDD IQDP I L PNMTEKFWEKSML T A WDL GKGDFR ILM ILKN STPEEL ATTFLEKFNTEVEDLFYQS SL ASWDYSTNIT, SKLAKTHQLDE IQDP I LPNMTEGF WEKSMLT A AWDLGKDFR IL M ILRN STPEEL ATTFLEKFNTEVEDLFYQS SL A WDYSTNIT SKLAKTHQLDE IQDP I LPNMTEGFWEKSML TANDLGKDFR ILM LRN

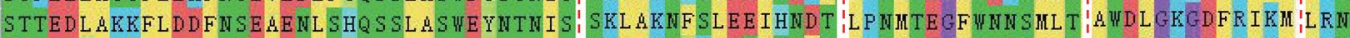

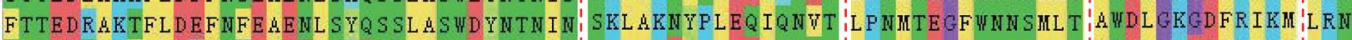

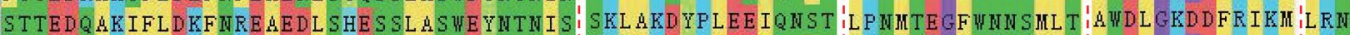

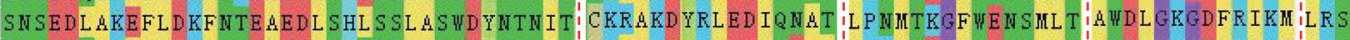

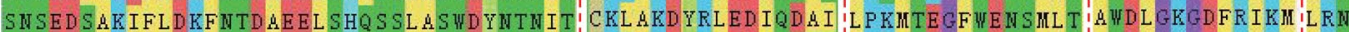

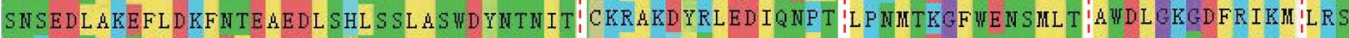

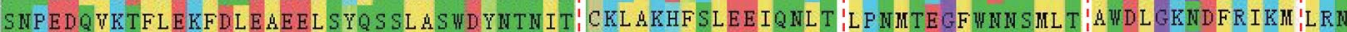

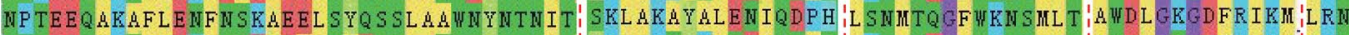

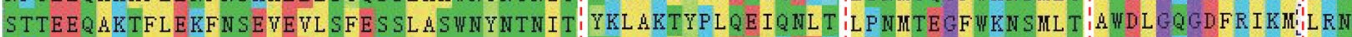

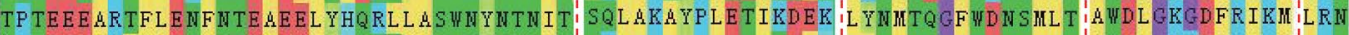

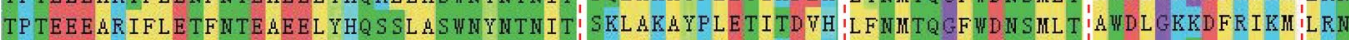

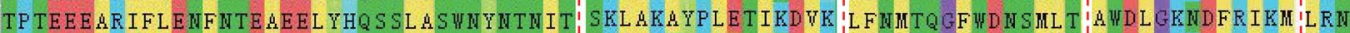

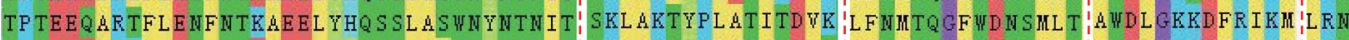

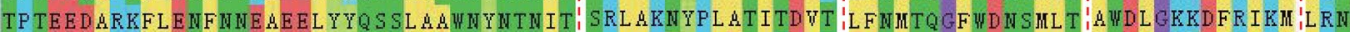

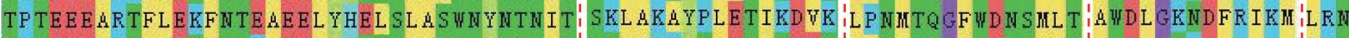
TP TEEEARTFLENFNTKAEEL YHQS SL ASWNYNTNIT SQLAKKY SLDT ITD DQ L FNMTQGFWDNSML T IAWDLGNKDFRIKM ILRN

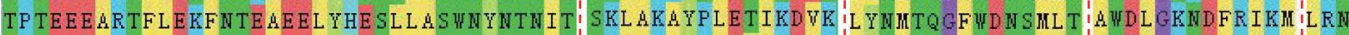

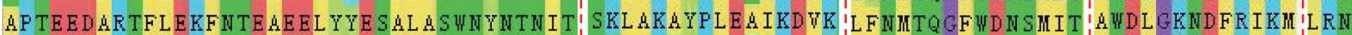

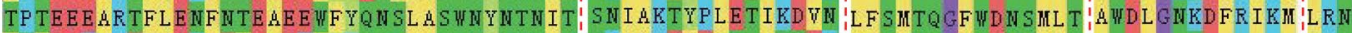

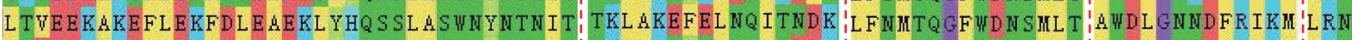

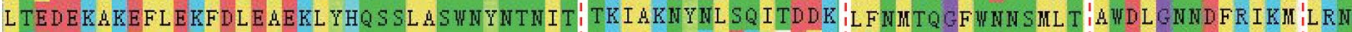

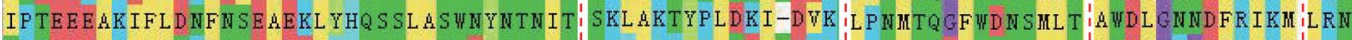

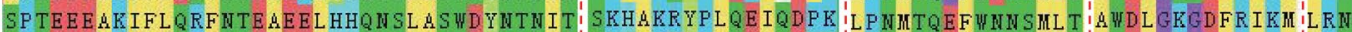

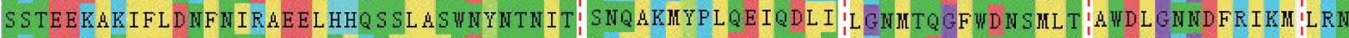
SP SEEKATIFLENFN SQAEDLSFKSALAAWDYNTNIT' SKLAKIYPLEE IQNAS 'LPNMTEGFWNNSML T, ANDLGKGDFR IKM 'LRN

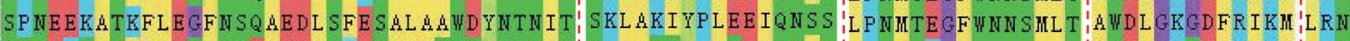
STTEKNAT IFLENFN SEAEDLSHE SALASWNYMTNIT: SKLAQTYPLQE IQNL T LP SMTPGFWNNSML T, AWDL GKNDFR IKM ILRN

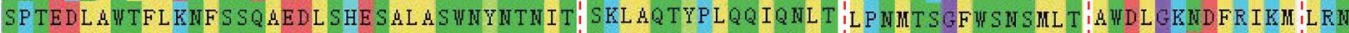

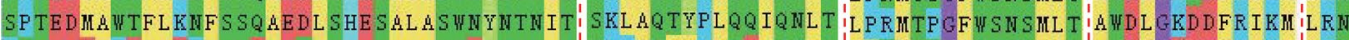

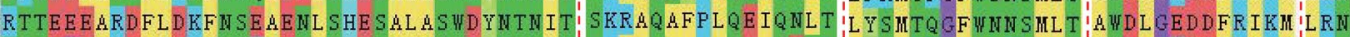
STTEGEAREFL DKFN SKAENL SHE SAL ASWDYNTNIT:SKLAQAFPLQE IQD ST LRNMT GF WNKSML T AWDL GKDDFR IKM LRN STIEEQATTFLENFNSKAEDLSHE SALAAWDYNTNIT:SKLAQTYPLQEIQNTT LPNMT SGFWDNSMLT,AWDLGKGDFRIKM,LRN

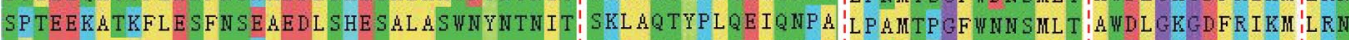

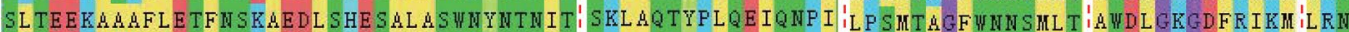

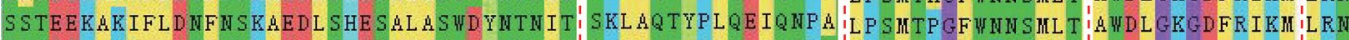

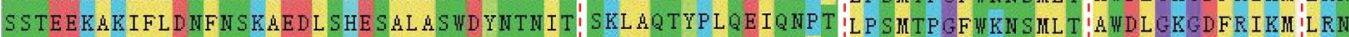

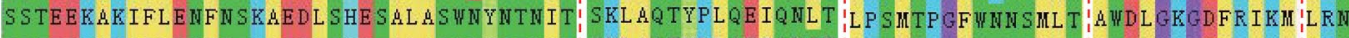
SSTEEKAK IF LENFN SKAEDLSHE SAL ASWNYNTNIT: SKLAQTYPLQEIQNST LP SMTPGFWNSMLT A WDLGKGDFRIKM LRN

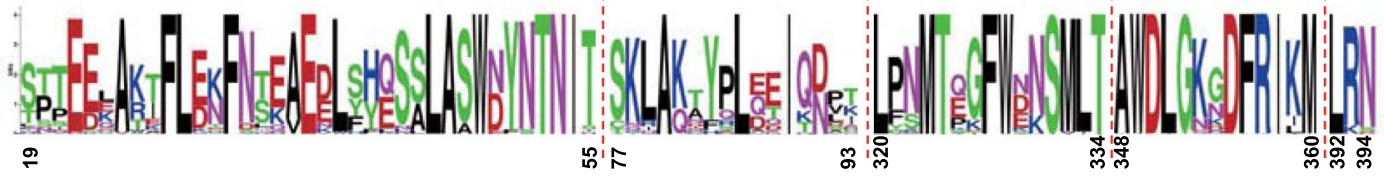

Extended Data Fig. 1 | Sequence alignment of RBD interacting region on $\mathbf{4 6}$ bat ACE2 orthologs. The alignment of RBD interaction regions were generated by MEGA-X software. Amino acids were coloured based on their residue features. The residues involved in the interaction between human ACE2 and SARS-CoV-2 RBD were indicated on the top. Sequence logo of the corresponding regions was generated by WebLogo (bottom). 
(1)

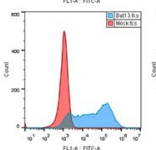

$\int_{-\infty}^{1}$

(1)

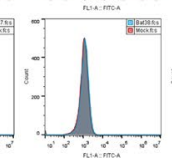

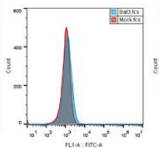
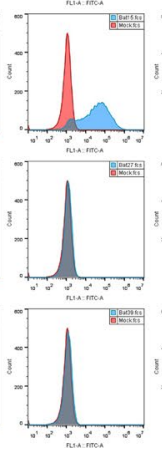
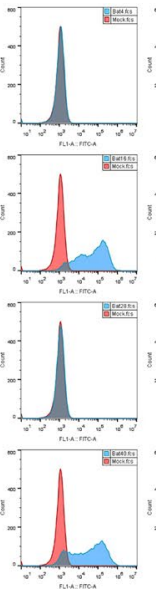
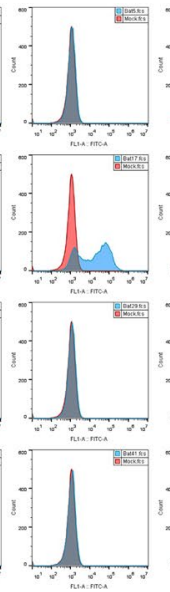
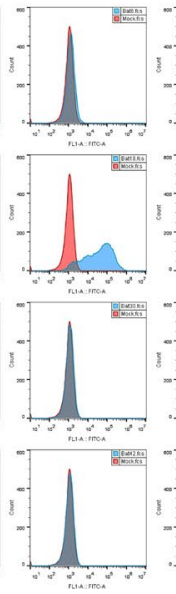
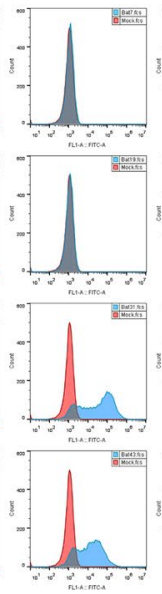
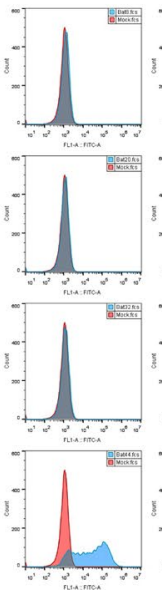
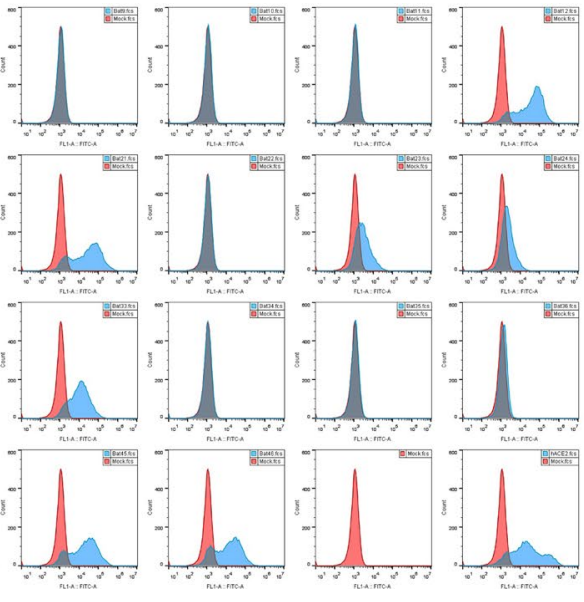

Extended Data Fig. 2 | Flow cytometry analysis of the SARS-CoV-2 RBD-hFc binding to 293T cells expressing different bat ACE2 orthologs. $293 \mathrm{~T}$ cells expressing bat ACE2 orthologs were incubated with $5 \mu \mathrm{g} / \mathrm{ml}$ of recombinant SARS-CoV-2 RBD-hFc protein at $37^{\circ} \mathrm{C}$ for 1 hour, and then washed and incubated with a Alexa Fluor 488 conjugated secondary antibody recognizing human IgG Fc. Histogram charts were generated by FlowJo with mock cells (sample 47) as negative control. 

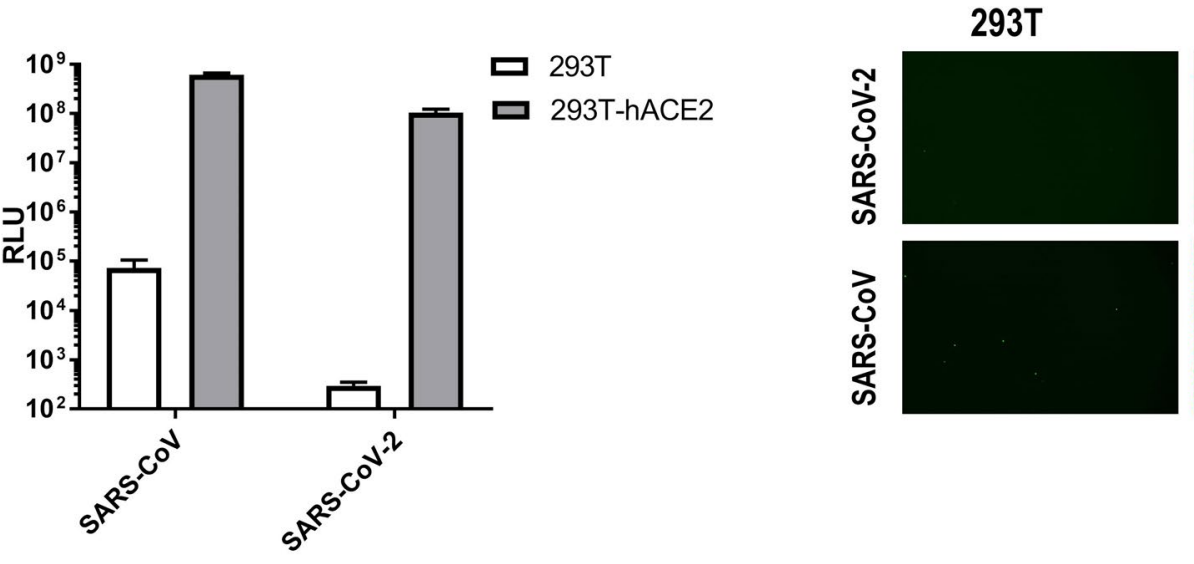

\section{T-hACE2}

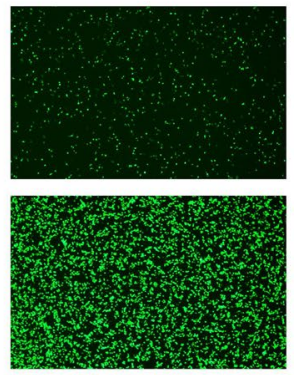

Extended Data Fig. 3 | Verification of SARS-CoV and SARS-CoV-2 pseudotypes on 293T cells expressing human ACE2. A high signal/background ratio of viral entry can be achieved on 293T-hACE2 cells for SARS-CoV and SARS-CoV-2 spike protein pseudotyped VSV-dG viruses expressing Firefly Luciferase (left) and GFP (right). 


\section{SARS-CoV-2 pseudotype entry}

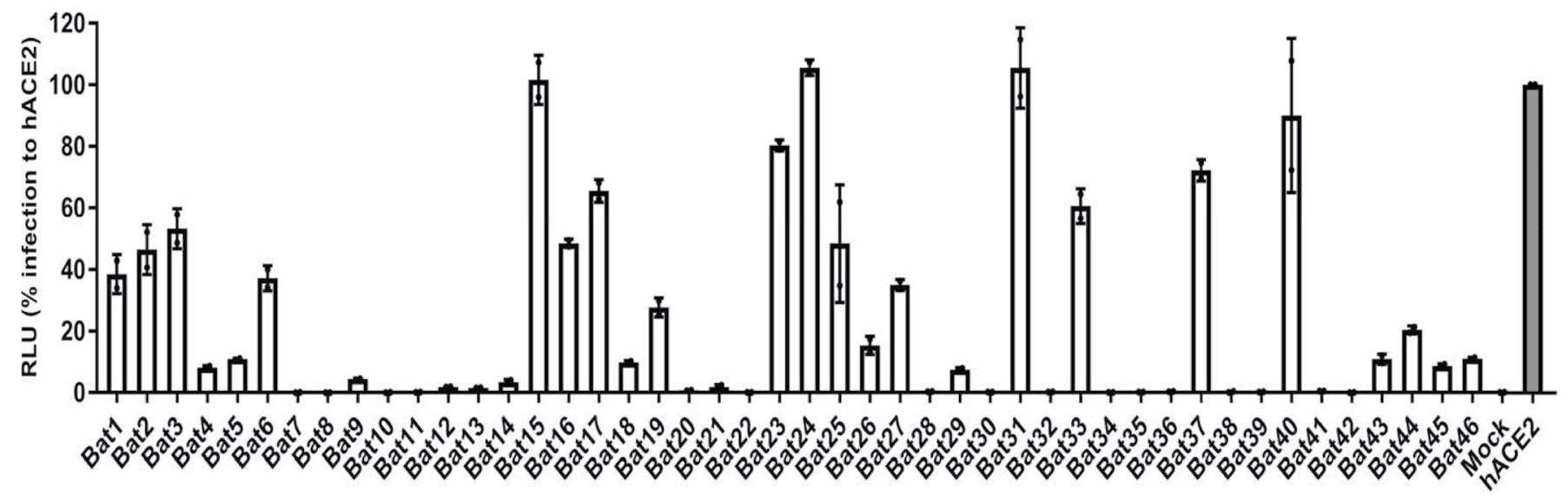

Extended Data Fig. 4 | Infection profile of virus pseudotyped with SARS-CoV-2 S protein without D614G variation. 293T cells expressing the indicated bats ACE2 orthologs were infected with SARS-CoV-2 (Wuhan-Hu-1 strain, without D614G variation) S protein pseudotyped VSV-dG-luc viruses. Luciferase units were determined at $20 \mathrm{hpi}$. Scale bar $=200 \mu \mathrm{m}$. The data are presented as the Mean \pm SD $(n=3)$. 


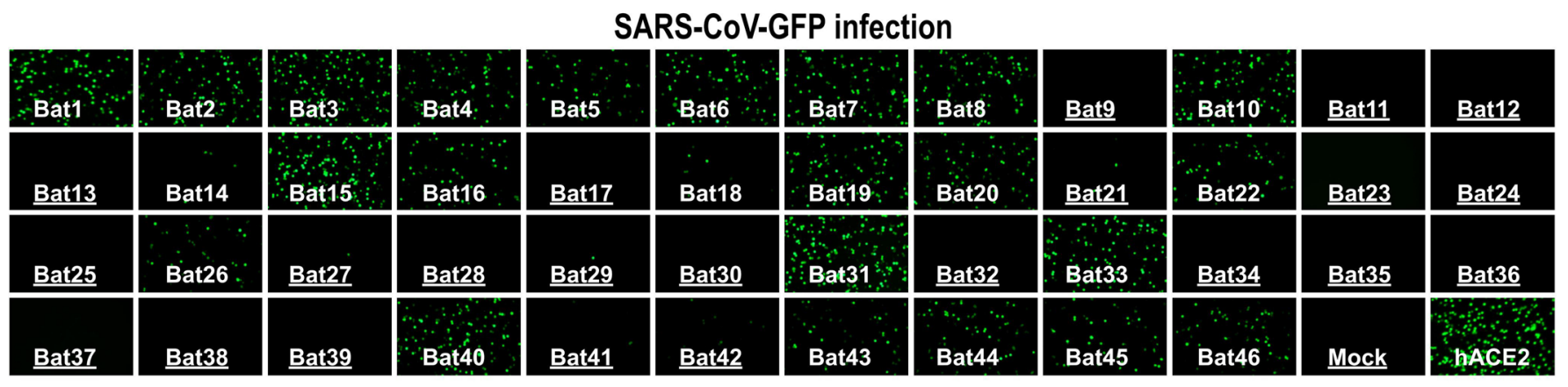

\begin{tabular}{|c|c|c|c|c|c|c|c|c|c|c|c|}
\hline Bat1 & Bat2 & Bat3 & Bat4 & Bat5 & Bat6 & Bat7 & Bat8 & Bat9 & Bat10 & Bat11 & Bat12 \\
\hline Bat13 & Bat14 & Bat15 & Bat16 & Bat17 & Bat18 & Bat19 & Bat20 & Bat21 & Bat22 & Bat23 & Bat24 \\
\hline Bat25 & Bat26 & Bat27 & Bat28 & Bat29 & Bat30 & Bat31 & Bat32 & Bat33 & Bat34 & Bat35 & Bat36 \\
\hline Bat37 & Bat38 & Bat39 & Bat40. & $3 a+41$ & Bat42 & Bat43 & Bat44 & Bat45 & Bat46 & Mock & hAGE2 \\
\hline
\end{tabular}

Extended Data Fig. 5 | The ability of bat ACE2 orthologs to support the entry of SARS-CoV and SARS-CoV-2 pseudotyped VSV-dG-GFP viruses. 293 T cells expressing the ACE2 orthologs of the indicated bats were infected with SARS-CoV and SARS-CoV-2 pseudotyped VSV-dG-GFP viruses. Images were captured at $20 \mathrm{hpi}$. Scale bar $=200 \mu \mathrm{m}$. 

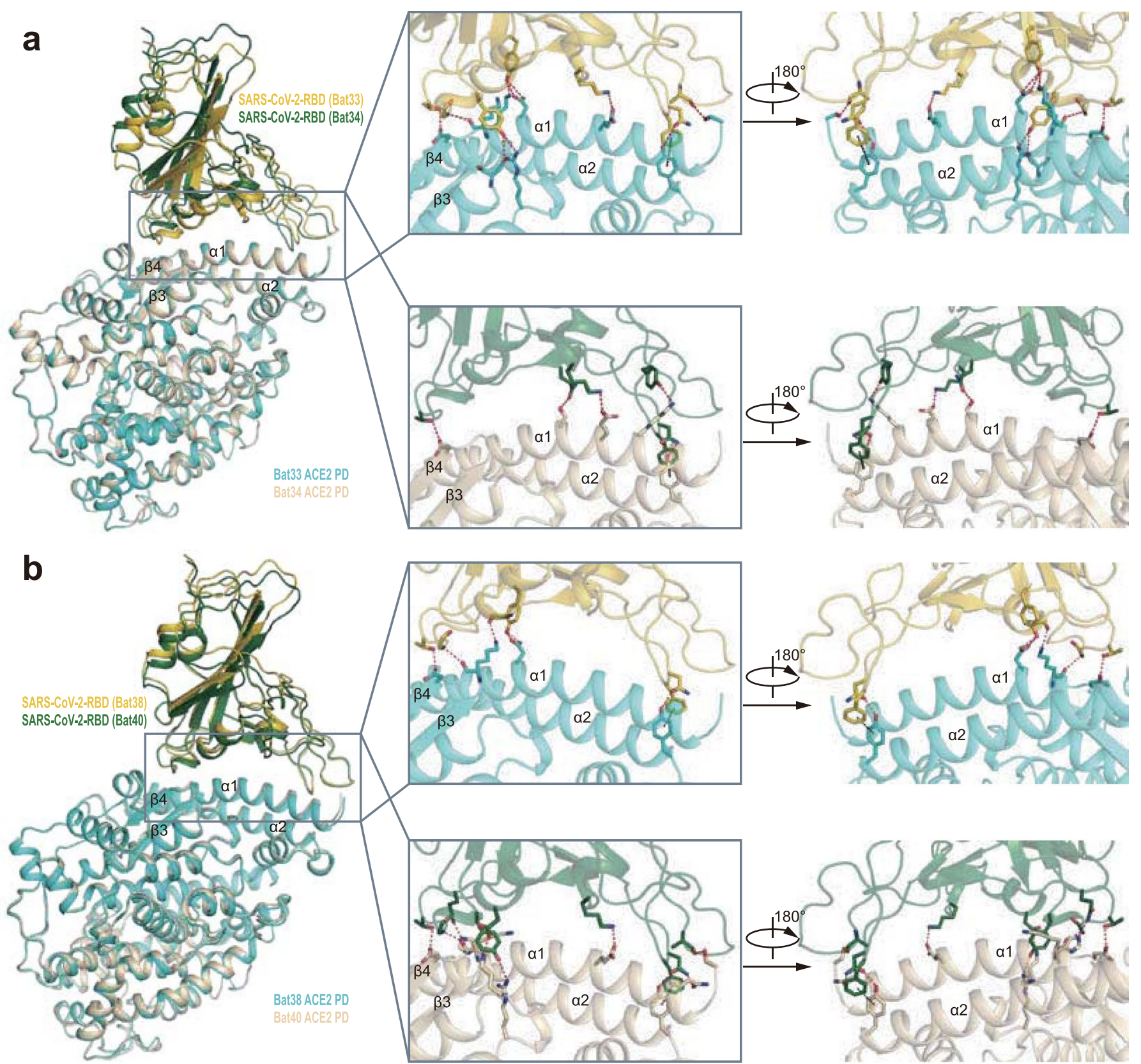

$134-12$

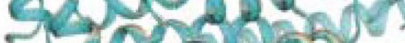

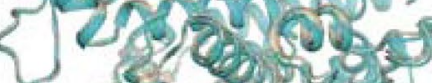

3 s. $20 y$

voustorns

cosio

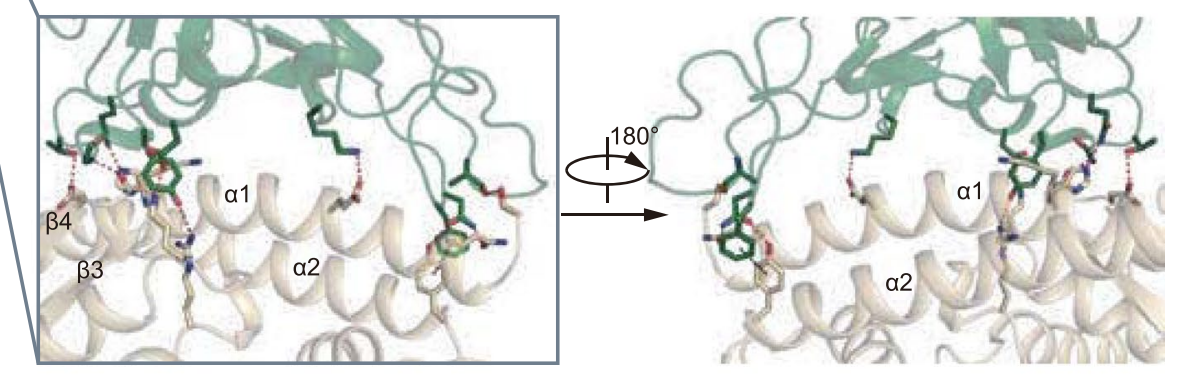

C
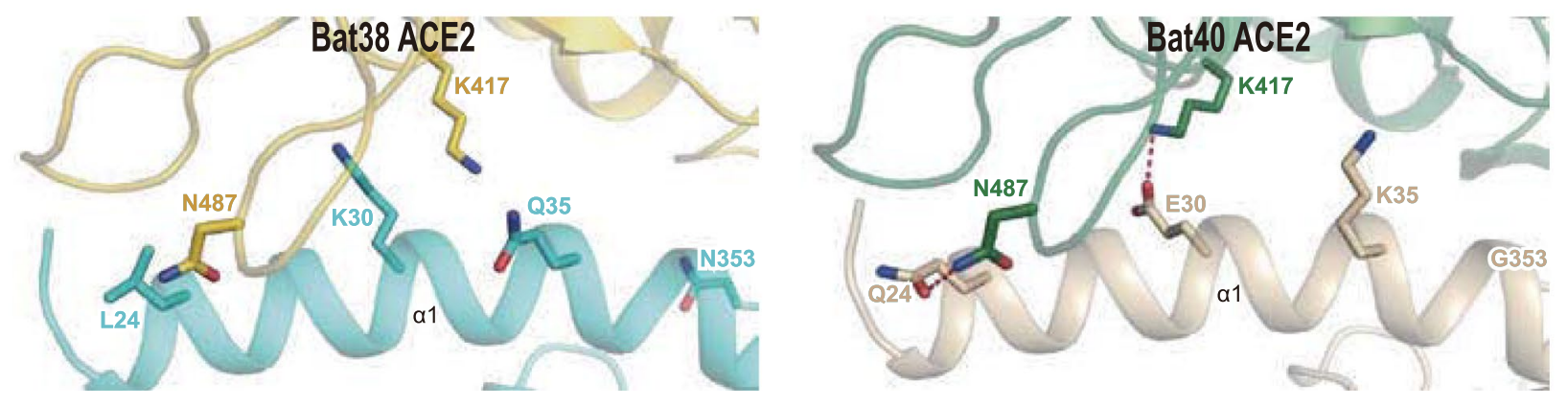

Extended Data Fig. 6 | See next page for caption. 
Extended Data Fig. 6 | Interface comparison between bat ACE2 with SARS-CoV-2-RBD. a, Structure alignment for the Bat33/SARS-CoV-2-RBD and Bat34/SARS-CoV-2-RBD. Bat33 and its complexed RBD are coloured cyan and gold, respectively; Bat34 and its complexed RBD are coloured wheat and green, respectively. The inset shows the interface of SARS-CoV-2-RBD with different ACE2. The residues which form hydrophilic interactions and aromatic interactions are shown as sticks. Here and subsequently, dotted lines between residues indicate interaction types: red for hydrogen bonds or ionic bonds and black for $\pi-\pi$ interactions. The right panel was obtained by clockwise rotation of the middle panel along a longitudinal axis. $\mathbf{b}$, Structure alignment for the Bat38/SARS-CoV-2-RBD and Bat40/SARS-CoV-2-RBD. Bat38 and its complexed RBD are coloured cyan and gold, respectively; Bat40 and its complexed RBD are coloured wheat and green, respectively. The inset shows the interface of SARS-CoV-2-RBD with different ACE2. The residues which form hydrophilic interactions and aromatic interactions are shown as sticks. The right panel was obtained by clockwise rotation of the middle panel along a longitudinal axis. c, Comparison of the interface between Bat38/SARS-CoV-2-RBD and Bat40/SARS-CoV-2-RBD. The mutated residues in ACE2 and the corresponding residues in RBD are shown and labelled. 
a

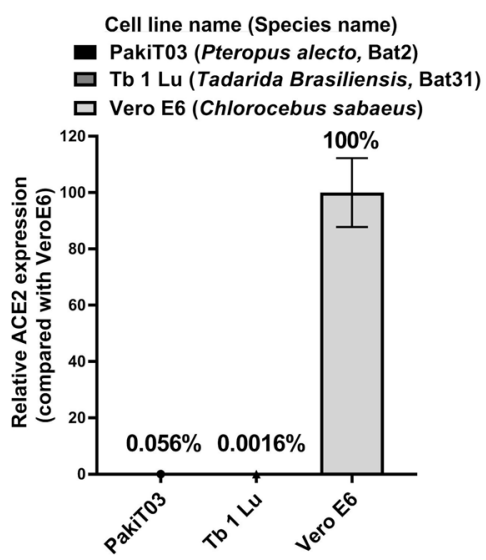

d

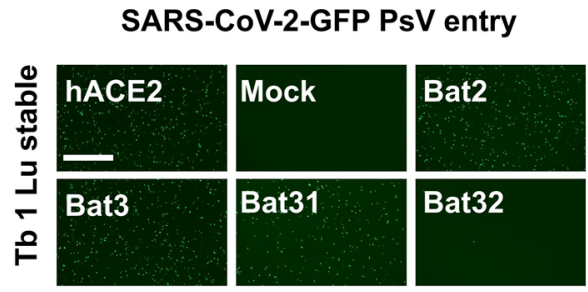

f

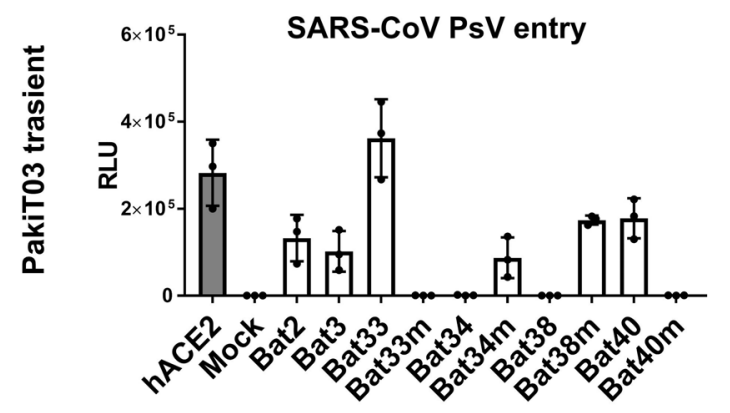

g

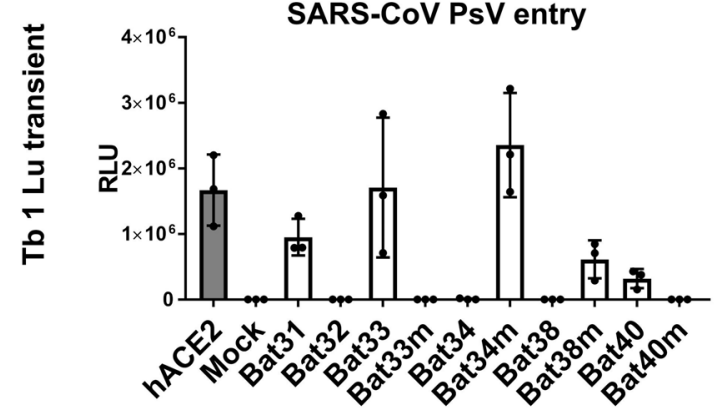

b

C

e
Lentiviral transduction efficiency

GFP
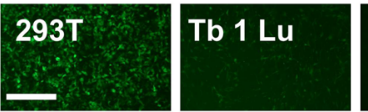

PakiT03

SARS-CoV PsV entry

SARS-CoV-2 PsV entry
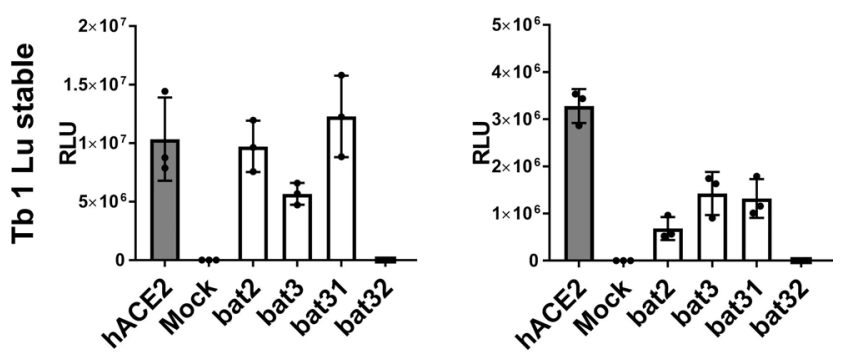

ACE2 orthologs expression level (anti-Flag)
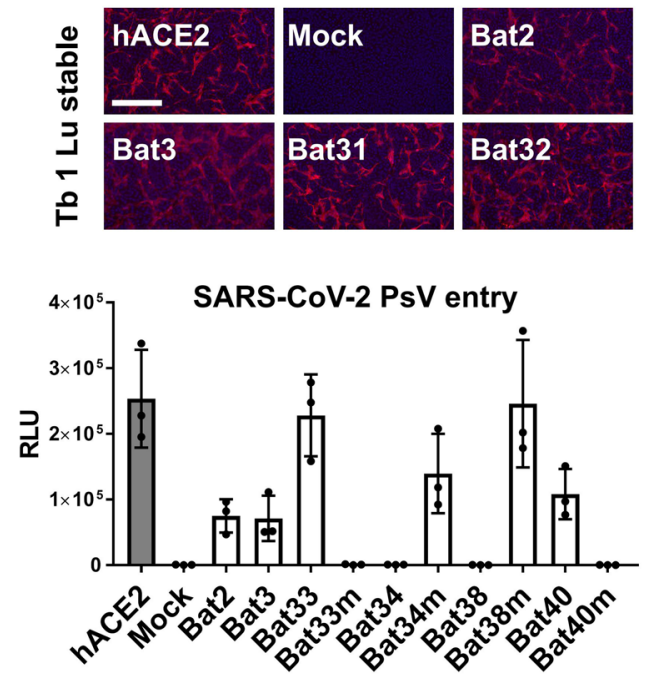

SARS-CoV-2 PsV entry

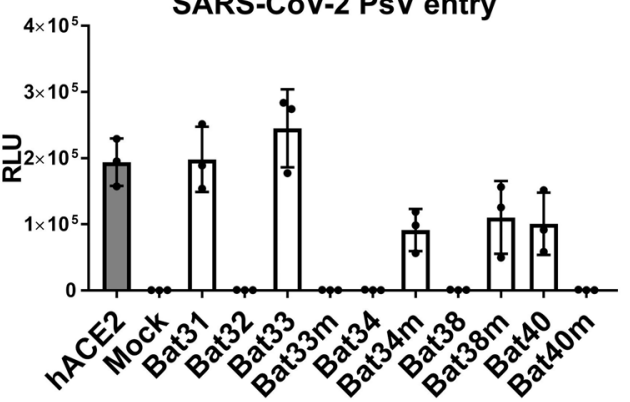

Extended Data Fig. 7 | ACE2 is the primary receptor for the species-specific entry of SARS-CoV and SARS-CoV- 2 in bat cell lines. a, The relative expression level of ACE2 orthologs in Vero-E6 and bat cell lines determined by Quantitative reverse transcription PCR(RT-qPCR). The data are presented as the Mean $\pm \operatorname{SEM~}(n=3)$. b, Lentiviral transduction efficiency of 293T (high), Tb 1 Lu (medium) and PakiT03 (very low). Cells were transduced with lentivirus carrying EGFP, and the images were captured at $72 \mathrm{~h}$ post-transduction. Tb $1 \mathrm{Lu}$ cell were selected for generating stable cell lines because of its higher transduction efficiency. c-e, The entry of SARS-CoV and SARS-CoV-2 pseudovirus into Tb 1 Lu cells stably expressing different ACE2 orthologs. The CoV entry efficiency was examined on Tb1-Lu stable-cells two weeks post lentiviral transduction (c,d). The data in c are presented as the Mean \pm SD $(n=3)$. The expression level of ACE2 orthologs was detected by immunofluorescence detecting the $C$ terminal fused Flag tags $(\mathbf{e})$. $\mathbf{f}-\mathbf{g}$, The entry of SARS-CoV and SARS-CoV-2 pseudovirus into bat cell lines transiently transfected with human and bat ACE2 orthologs. Scale bar $=200 \mu \mathrm{m}$. The data are presented as the Mean \pm SD $(n=3)$. 


\section{Reporting Summary}

Nature Research wishes to improve the reproducibility of the work that we publish. This form provides structure for consistency and transparency in reporting. For further information on Nature Research policies, see our Editorial Policies and the Editorial Policy Checklist.

\section{Statistics}

For all statistical analyses, confirm that the following items are present in the figure legend, table legend, main text, or Methods section.

n/a Confirmed

$\square \bigotimes$ The exact sample size $(n)$ for each experimental group/condition, given as a discrete number and unit of measurement

$\square$ \A statement on whether measurements were taken from distinct samples or whether the same sample was measured repeatedly

$\triangle$ The statistical test(s) used AND whether they are one- or two-sided

X $\square$ Only common tests should be described solely by name; describe more complex techniques in the Methods section.

Х $\square$ A description of all covariates tested

Х $\square$ A description of any assumptions or corrections, such as tests of normality and adjustment for multiple comparisons

$\triangle$ A full description of the statistical parameters including central tendency (e.g. means) or other basic estimates (e.g. regression coefficient)

$\bigotimes$ AND variation (e.g. standard deviation) or associated estimates of uncertainty (e.g. confidence intervals)

Х For null hypothesis testing, the test statistic (e.g. $F, t, r$ ) with confidence intervals, effect sizes, degrees of freedom and $P$ value noted

Wive $P$ values as exact values whenever suitable.

Х $\square$ For Bayesian analysis, information on the choice of priors and Markov chain Monte Carlo settings

$\bigotimes \square$ For hierarchical and complex designs, identification of the appropriate level for tests and full reporting of outcomes

Х $\square$ Estimates of effect sizes (e.g. Cohen's $d$, Pearson's $r$ ), indicating how they were calculated

Our web collection on statistics for biologists contains articles on many of the points above.

\section{Software and code}

Policy information about availability of computer code

Data collection Spectra MaxiD3 multi-well Luminometer (Molecular Devices, United States) and GloMax ${ }^{\circledR}$ 20/20 Luminometer (Promega, United States) for measuring the luciferase activity. CytoFLEX Flow Cytometer (Beckman Coulter, United States) for generating the flow cytometry data

Data analysis GraphPad Prism 7 was used in this study. FlowJo version 10 was used for analyzing flow cytometry data

For manuscripts utilizing custom algorithms or software that are central to the research but not yet described in published literature, software must be made available to editors and reviewers. We strongly encourage code deposition in a community repository (e.g. GitHub). See the Nature Research guidelines for submitting code \& software for further information.

\section{Data}

Policy information about availability of data

All manuscripts must include a data availability statement. This statement should provide the following information, where applicable:

- Accession codes, unique identifiers, or web links for publicly available datasets

- A list of figures that have associated raw data

- A description of any restrictions on data availability

The authors declare that all the data supporting the findings of this study are available within the paper and its supplementary information files. All the raw data underlying Figures and Tables are available upon request 


\section{Field-specific reporting}

Please select the one below that is the best fit for your research. If you are not sure, read the appropriate sections before making your selection. \Life sciences $\square$ Behavioural \& social sciences Ecological, evolutionary \& environmental sciences

For a reference copy of the document with all sections, see nature.com/documents/nr-reporting-summary-flat.pdf

\section{Life sciences study design}

All studies must disclose on these points even when the disclosure is negative.
Sample size
We analyzed 46 bat ACE2 sequences as well as human ACE2 sequence in our study
Data exclusions
There is no data excluded from the analysis
Replication
Luciferase assays were conducted with three biological replicates, each with two-three technical replicates.
Randomization
In this study, we included as many bat ACE2 sequences as possible. No grouping is applied
Blinding
No grouping is applied. Therefore blinding is not applicable here

\section{Reporting for specific materials, systems and methods}

We require information from authors about some types of materials, experimental systems and methods used in many studies. Here, indicate whether each material, system or method listed is relevant to your study. If you are not sure if a list item applies to your research, read the appropriate section before selecting a response.

\begin{tabular}{l|l} 
Materials \& experimental systems \\
\hline $\mathrm{n} / \mathrm{a}$ & Involved in the study \\
\hline & $\bigotimes$ Antibodies \\
$\square$ & $\square$ Eukaryotic cell lines \\
$\square$ & $\square$ Animaeontology and archaeology \\
$\square$ & $\square$ Human research participants \\
$\square$ & $\square$ Clinical data
\end{tabular}

\begin{tabular}{l|l}
\multicolumn{2}{l}{ Methods } \\
\hline n/a & Involved in the study \\
$\square$ & $\square$ ChIP-seq \\
$\square$ & $\bigotimes$ Flow cytometry \\
$\searrow$ & $\square$ MRI-based neuroimaging
\end{tabular}

\section{Antibodies}

Antibodies used

Flag tag (9A3, \#8146S, Cell signaling technology, United States); Secondary goat anti-mouse antibody conjugated with Alexa Fluor 594 (A11032, Thermo Fisher Scientific, United States); Alexa Fluor 488 conjugated Goat anti-Human IgG (A11013, Thermo Fisher Scientific, United States)

Validation

$$
\text { Vendor validated }
$$

\section{Eukaryotic cell lines}

Policy information about cell lines

Cell line source(s)

Authentication

Mycoplasma contamination

Commonly misidentified lines

(See ICLAC register)
HEK293T cells (293T, ATCC, CRL-3216); Tb 1 Lu cells (ATCC, CCL-88); PakiT03 cells (a gift from professor Peng Zhou); VERO-E6 cells (ATCC, CRL-1586); 11-Hybridoma (ATCC, CRL-2700)

Not authenticated

Cell lines used in this study were not tested for mycoplasma contamination

No commonly misidentified line was used 


\section{Plots}

Confirm that:

邓 The axis labels state the marker and fluorochrome used (e.g. CD4-FITC).

$\bigotimes$ The axis scales are clearly visible. Include numbers along axes only for bottom left plot of group (a 'group' is an analysis of identical markers).

$\square$ All plots are contour plots with outliers or pseudocolor plots.

$\square$ A numerical value for number of cells or percentage (with statistics) is provided.

\section{Methodology}

Sample preparation

The 293T cells stably overexpressing different ACE2 orthologs were incubated with RBDs and antibody in two consecutive staining steps. The first was an incubation with SARS-CoV-RBD-hFc or SARS-CoV-2-RBD-hFc (5-10 $\mu \mathrm{g} / \mathrm{ml})$ in DMEM with $2 \%$ FBS for 1 hour at $37^{\circ} \mathrm{C}$. The second utilized an Alexa Fluor 488 conjugated Goat anti-Human IgG ( A11013, Thermo Fisher Scientific, United States) in $2 \mu \mathrm{g} / \mathrm{ml}$ for additional $30 \mathrm{~min}$ at $37^{\circ} \mathrm{C}$. The stained cells were detached by $5 \mathrm{mM}$ EDTA/PBS before strained through a $70 \mu \mathrm{m}$ cell mesh, and then analyzed with a CytoFLEX Flow Cytometer (Beckman Coulter, United States). More information are available on Methods sections

Instrument

Software

Cell population abundance

Gating strategy
CytoFLEX Flow Cytometer (Beckman Coulter, United States)

FlowJo version 10

We did not count the cell population percentage

The RBD-hFc binding 293T cells were gated as Alexa Fluor 488 conjugated Goat anti-Human IgG+ single cells. More information are available on Methods sections

Tick this box to confirm that a figure exemplifying the gating strategy is provided in the Supplementary Information. 